\title{
Microscopic Transport Theory of Nuclear Processes
}

\author{
K. Dietrich ${ }^{1}$, J.-J. Niez, J.-F. Berger \\ CEA, DAM, DIF, F-91297, Arpajon, France
}

\begin{abstract}
We formulate a microscopic theory of the decay of a compound nucleus through fission which generalizes earlier microscopic approaches of fission dynamics performed in the framework of the adiabatic hypothesis. It is based on the constrained Hartree-Fock-Bogoliubov procedure and the Generator Coordinate Method, and requires an effective nucleon-nucleon interaction as the only input quantity. The basic assumption is that the slow evolution of the nuclear shape must be treated explicitely, whereas the rapidly time-dependent intrinsic excitations can be treated by statistical approximations. More precisely, we introduce a "reference density" $\rho_{P}$ which represents the slow evolution of the nuclear shape by a reduced density matrix and the state of intrinsic excitations by a canonical distribution at each given shape of the nucleus. The shape of the nuclear density distribution is described by parameters ("generator coordinates" q), not by "superabundant" degrees of freedom introduced in addition to the complete set of nucleonic degrees of freedom.

We first derive a rigorous equation of motion for the reference density $\rho_{P}$ and, subsequently, simplify this equation on the basis of the "Markov approximation". The temperature $T$ which appears in the canonical distribution is determined by the requirement that, at each time $t$, the reference density should correctly reproduce the mean excitation energy at given values of the shape parameters $q$. The resulting equation for the "local" temperature $T(q, t)$ must be solved together with the equations of motion obtained for the reduced density matrix $R\left(q_{1}, q_{2} ; t\right)$.
\end{abstract}

Key words: Nuclear transport theory, fission, HFB, GCM

PACS: , 21.60.-n, 21.60.Jz, 24.10.Pa, 24.60.-k, 24.75.+i

\section{Introduction}

There are many systems in nature which are characterized by more than one time scale. Let us assume, for simplicity, that there is one time-scale for the slow, usually collective motion (described by variables or parameters $q$ ) and one much shorter time-scale for the rapid motion of a large number of individual particles (with the degrees of freedom $x$ ). Whereas the slow motion must be treated explicitly, the rapid dynamical evolution can often be approximated by a thermodynamical or, more generally, by some statistical "ansatz". There is a vast literature on the theoretical description of such systems. One refers to them as "transport theories".

Their intense study started in the $19^{\text {th }}$ century with the observation of the "Brownian motion" [1], i.e. the motion of a pollen or dust particle submerged in a medium of the randomly moving molecules of a gas or of a liquid. As an average result of the randomly distributed collisions, the "Brownian particles" are exposed to a "friction force" $-\gamma \overline{\vec{v}(t)}$ reducing their average velocity $\overline{\vec{v}(t)}$, and to a dissipative force measured by a parameter $D$. The dissipation parameter $D$ is determined by the time rate $\frac{d}{d t} \overline{\left(\vec{r}(t)-\overline{\vec{r}(t))^{2}}\right.}$ of the mean square deviation of the trajectories $\vec{r}(t)$ from the average trajectory $\overline{\vec{r}(t)}$, the mean values being performed over an ensemble of trajectories.

It was for the model of Brownian motion that Einstein derived the simple classical relation [2] :

$$
\gamma=\frac{k_{B} T}{M D}
$$

\footnotetext{
${ }^{1}$ Permanent address : Physics Department, Technische Universität München, 85747 Garching, Germany Preprint submitted to Elsevier
} 
( $M=$ mass of the Brownian particle, $T=$ temperature, $k_{B}=$ Boltzmann constant). The generalized quantum-mechanical version of the Einstein relation (1.1) is called "fluctuation-dissipation theorem" [3]. It will be discussed in Section 5 of our manuscript.

The standard form of transport theories is based on a Hamiltonian $\hat{H}$ of the form [4, 5]

$$
\hat{H}=\hat{H}_{S}(q)+\hat{H}_{B}(x)+\hat{H}_{S B}(x, q)
$$

$\hat{H}_{S}$ depends on the slowly time-dependent variables $q, \hat{H}_{B}$, often referred to as "bath Hamiltonian", depends on the rapidly time-dependent variables $x$, and the Hamiltonian $\hat{H}_{S B}$ represents the coupling between both kinds of degrees of freedom.

In the case of the atomic nucleus and numerous other systems, the complete set of independent degrees of freedom is given by the rapidly time-dependent variables of the constituent particles only. Here, the slow part of the time evolution is produced by a slow collective component of the particle motion. As an example, a nuclear fission or fusion-fission process proceeds through the slowly varying shape of the nuclear density distribution.

If, nevertheless, one chooses to describe such a system by a Hamiltonian of type (1.2), the shape variables $q$ are not independent of the particle variables $x$ but related to them by constraints. Thus, for establishing a Hamiltonian of type (1.2), one needs a theory of collective motion as a prerequisite. Usually, one adopts a method proposed by Bohm and Pines [6]. It is based on the constraint that the collective variables $q$ are to be equal to mean values of certain multipole operators $\hat{q}(x)$, which depend on the variables $x$ of the individual particles :

$$
q=\operatorname{Tr}(\hat{q}(x) \hat{\rho})
$$

Here, $\hat{\rho}$ is the density operator of the system or some approximation of it. Strictly speaking, fluctuations around these mean values exceed the scope of this approach.

We, henceforth, consider in particular the nuclear fission process. One of the intriguing aspects of this process is the appearance of large fluctuations [7] of quantum mechanical as well as thermodynamical origin, as exemplified by the large widths of the mass- and kinetic energy-distributions observed even at small excitation energies of the fissioning system.

In order to remedy this difficulty of the existing dynamical fission theories, we apply the Generator Coordinate Method (GCM) which has been introduced by Hill and Wheeler in their well-known paper of 1953 [8]. In this method, the wavefunction $\Psi\left(x_{1}, \ldots, x_{A} ; t\right)$ of the nucleus is expanded in terms of a set of basis functions $\phi_{a}\left(x_{1}, \ldots, x_{A} ; q\right)$ according to :

$$
\Psi\left(x_{1}, \ldots, x_{A} ; t\right)=\sum_{a} \int d q f_{a}(q, t) \phi_{a}\left(x_{1}, \ldots, x_{A} ; q\right)
$$

The wave-functions $\phi_{a}\left(x_{1}, \ldots, x_{A} ; q\right)$ depend on the nucleon variables $x_{1}, \ldots, x_{A}$ and on a limited number of "generator coordinates" (g.c.) $q$ which serve to describe the possible shapes of the nucleus. The subscript " $a$ " defines the quantum states of intrinsic excitation which may occur for each given shape $q$. The important aspect of the ansatz (1.4) is that the shape parameters $q$ are summed over. Thus, they do not represent "over-abundant" variables.

So far, in actual applications of the GCM to nuclear fission and to nuclear collective excitations, only the ground states $\phi_{a_{0}}\left(x_{1}, \ldots, x_{A} ; q\right)$ of the Hartree-Fock-Bogoliubov (HFB) method have been superimposed in [1.4] [9, 10, 11]. These ground states are defined as the solutions of the variational problem

$$
\delta\left\langle\phi_{a_{0}}(q)\left|\hat{H}^{\prime}\right| \phi_{a_{0}}(q)\right\rangle \equiv \delta\left\langle\phi_{a_{0}}(q)\left|\hat{H}-\lambda \hat{q}-\mu_{n} \hat{N}-\mu_{p} \hat{Z}\right| \phi_{a_{0}}(q)\right\rangle=0
$$

In Eq. 1.5], the quantities $\hat{H}, \hat{q}, \hat{N}$ and $\hat{Z}$ represent the total Hamiltonian, a set of multipole operators, the neutron- and proton-number operators, respectively. The Lagrange parameters $\lambda, \mu_{n}$ and $\mu_{p}$ are defined by the constraints

$$
\begin{aligned}
& \left\langle\phi_{a_{0}}(q)|\hat{q}| \phi_{a_{0}}(q)\right\rangle=q \\
& \left\langle\phi_{a_{0}}(q)|\hat{N}| \phi_{a_{0}}(q)\right\rangle=N \\
& \left\langle\phi_{a_{0}}(q)|\hat{Z}| \phi_{a_{0}}(q)\right\rangle=Z
\end{aligned}
$$

where $N$ and $Z$ are the neutron and proton numbers of the nucleus, and $q$ is a set of generator coordinates. As usual in the constrained HFB method, the Hamiltonian $\hat{H}$ appearing in 1.5 must be built using an effective nucleon-nucleon 
interaction adapted to the description of the intrinsic structure of the mean-field states $\phi_{a_{0}}(q)$, such as the Skyrme [12] or the Gogny [13] effective interactions. In Refs. [9, 10, 11], the Gogny force, which is known to provide a good description of both the average nuclear mean field and pairing correlations was used.

Omitting intrinsic excitations $\phi_{a}(q) \neq \phi_{a_{0}}(q)$ in Eq. (1.4) implies that only low-lying vibrational excitations of the system are taken into account on the way to fission or fusion. Nevertheless, many aspects of the low-energy fission process like the coexistence of a fusion and a fission valley [9] and the basic features of the fragment kinetic energy distribution [10] have been rather successfully obtained in spite of this restriction.

In our present work, we want to go beyond these calculations in essentially two ways :

(i) We include intrinsic excitations, i.e. our Hill-Wheeler basis derived from constrained HFB calculations includes intrinsic excitations $\phi_{a}(q)$ up to a given upper energy limit.

(ii) We represent the system by the density operator $\hat{\rho}(t)$ instead of describing it by a single state vector $\Psi(t)$.

As excited states $\phi_{a}(q)$, we use the eigenstates of the one-body part $\hat{H}_{1}^{\prime}(q)$ in the Wick decomposition of the Hamiltonian $\hat{H}^{\prime}(q)$ (see Eq. 1.10$)$ ).

As the part $\hat{H}^{\prime(20)}+\hat{H}^{(02)}$ of the Wick decomposition involving the creation $\left(\hat{H}^{(20)}\right)$ or annihilation $\left(\hat{H}^{\prime(02)}\right)$ of two quasi-particles vanishes as a result of the variational principe $(1.5)$, the Hamiltonian $\hat{H}^{\prime}(q)$ reads :

$$
\hat{H}^{\prime}(q)=H^{(0)}(q)+\hat{H}^{(11)}(q)+\hat{H}_{(2)}^{\prime}(q)
$$

The Hamiltonian $\hat{H}^{(11)}(q)$ comprises all the terms with one creation and one annihilation operator, and $\hat{H}_{(2)}^{\prime}(q)$ is defined as the sum of all terms involving four quasi-particle creation or annihilation operators in normal order. Hence:

$$
\hat{H}_{1}^{\prime}(q) \phi_{a}(q) \equiv\left(H^{\prime(0)}(q)+\hat{H}^{\prime(11)}(q)\right) \phi_{a}(q)=E_{1 a}^{\prime}(q) \phi_{a}(q)
$$

The "vacuum energy" at a given $q$ is given by

$$
E_{1 a_{0}}^{\prime}(q)=H_{(0)}^{\prime}(q)
$$

with the corresponding vacuum state $\phi_{a_{0}}(q)$ (see Eq. (1.5)). A presumably preferable choice of $\phi_{a_{0}}(q)$ would be provided by the solutions of the RPA at a given value of the g.c. $q$. Given the fact that it is difficult to determine these multiphonon states for a heavy nucleus, we shall use the states defined in Eq. (1.10) which represent multi-quasiparticle states in a nucleus with the mean multipole moments $q$.

Two well-known practical difficulties are inherent to the GCM :

(i) The Hill-Wheeler states $\phi_{a}(q)$ are not orthogonal to each other.

(ii) There may occur a number of linearly dependent or almost linearly dependent states among the basis states $\phi_{a}(q)$. This is revealed by the fact that the overlap matrix

$$
N_{a_{1} a_{2}}\left(q_{1}, q_{2}\right):=\left\langle\phi_{a_{1}}\left(q_{1}\right) \mid \phi_{a_{2}}\left(q_{2}\right)\right\rangle
$$

may have a number of vanishing or almost vanishing eigenvalues.

We apply two remedies to cope with these difficulties:

1) We work with discrete values of the generator coordinates (g.c.) instead of the continuous range of values implied in Eq. (1.4): Each given type of g.c. $q$ varying from a lower limit in $f(q)$ to an upper limit $\sup (q)$ is replaced by a finite number of discrete values with a spacing $\Delta q$ :

$$
q \rightarrow \inf (q)+n \Delta q \leq \sup (q)
$$

where the positive integer numbers $n$ range from 0 to $(\sup (q)-\operatorname{in} f(q)) / \Delta q$. The step length $\Delta q$ must be chosen in a physically reasonable way

$$
\int d q \rightarrow \Delta q \sum_{n} \equiv \sum_{q}
$$


As we incorporate intrinsic excitations "a" for each set of the g.c. $q$, we surmise that the required accuracy of description can be achieved with not too small values of the step length $\Delta q$.

2) We either use a biorthogonal system of basis states $\widetilde{\phi_{a}(q)}, \phi_{a}(q)$ with

$$
\left\langle\widehat{\phi_{a_{1}}\left(q_{1}\right)} \mid \phi_{a_{2}}\left(q_{2}\right)\right\rangle=\delta_{a_{1} a_{2}} \delta_{q_{1} q_{2}}
$$

or we replace the non-orthogonal Hill-Wheeler states $\phi_{a}(q)$ by a system of orthonormal basis functions $\psi_{a}(q)$.

Our paper is organized as follows:

The just mentioned alternative of either using "adjoint" (=“dual") basis states $\widetilde{\phi_{a}(q)}$ as "bra-states" in all the matrix elements or of introducing an adequately chosen orthonormal set of basis states $\psi_{a}(q)$ will be dealt with in Section 2 . In Section 3, we shall introduce our basic approximation $\hat{\rho}_{P}(t)$ for the statistical operator $\hat{\rho}(t)$ of the total system and derive a rigorously valid equation of motion for $\hat{\rho}_{P}(t)$ using the formalism introduced by Nakajima and Zwanzig [14, 15]. This rigorously valid equation cannot be solved as it stands. An important and well-known step in transport theories is the application of the so-called "Markov approximation". Applying this approximation, we arrive at an integro-differential equation for $\hat{\rho}_{P}(t)$ which is amenable to a numerical solution.

The physical content of the Markov approximation is that the slowly time-dependent part $\hat{\rho}_{P}(t)$ of the statistical operator $\hat{\rho}(t)$, which is designed to describe the slow collective part of the time evolution, should only depend in a negligible way on the detailed phase relations of the rapidly time-dependent intrinsic variables. The detailed form of the Markov approximation will be treated in Section 3 .

In the same Section, we shall also introduce a decomposition of the total Hamiltonian $\hat{H}$ into an average collective potential $\hat{H}_{c o l l}$ and a remainder $\hat{H}_{c p l}=\hat{H}-\hat{H}_{c o l l}$, and we shall postulate that the coupling Hamiltonian $\hat{H}_{c p l}$ can be treated in perturbation theory.

Within the slowly time-dependent part $\hat{\rho}_{P}(t)$ of the density operator $\hat{\rho}(t)$, the distribution of the system over the intrinsic excitations at given value of the g.c. $q$ will be described by a canonical distribution which depends on a temperature $T$. This temperature $T(q, t)$, which should be considered as a useful parameter, will be chosen as a function of the generator coordinate $q$ and of the time $t$ in such a way that the ansatz $\hat{\rho}_{P}(t)$ represents optimally the slowly time-dependent part of the density operator $\hat{\rho}(t)$. The choice of the temperature $T(q, t)$ and the equation by which it is to be determined will be presented in Section 4

It is a well-known feature of the Generator Coordinate Method (GCM) that matrix-elements of the Hamiltonian or of other observables $\hat{O}$ between Hill-Wheeler states $\phi_{a_{1}}\left(q_{1}\right)$ and $\phi_{a_{2}}\left(q_{2}\right)$ contain a factor which depends in a narrow, approximately Gaussian form on the difference $r=q_{1}-q_{2}$, whereas the remaining part of the matrix element is a smooth function of the g.c. $q_{1}$ and $q_{2}$. Writing the elements of the overlap matrix in the form

$$
N_{a_{1} a_{2}}\left(q_{1}, q_{2}\right)=\left\langle\phi_{a_{1}}\left(q_{1}\right) \mid \phi_{a_{2}}\left(q_{2}\right)\right\rangle=e^{-r^{2} / 2 \sigma^{2}} n_{a_{1} a_{2}}\left(q_{1}, q_{2}\right),
$$

the factor $n_{a_{1} a_{2}}\left(q_{1}, q_{2}\right)$ depends smoothly on the variables $q_{1}$ and $q_{2}$. Analogously, it is useful to define a "reduced matrix element" $\left\langle\phi_{a_{1}}\left(q_{1}\right)|\hat{o}| \phi_{a_{2}}\left(q_{2}\right)\right\rangle$ by the equation

$$
\left\langle\phi_{a_{1}}\left(q_{1}\right)|\hat{o}| \phi_{a_{2}}\left(q_{2}\right)\right\rangle:=\frac{\left\langle\phi_{a_{1}}\left(q_{1}\right)|\hat{O}| \phi_{a_{2}}\left(q_{2}\right)\right\rangle}{N_{a_{1} a_{2}}\left(q_{1}, q_{2}\right)}
$$

It is a convenient and usually good approximation [16] to assume that the reduced matrix-elements $\left\langle\phi_{a_{1}}\left(q_{1}\right)|\hat{o}| \phi_{a_{2}}\left(q_{2}\right)\right\rangle$ $\equiv\left\langle\phi_{a_{1}}\left(q_{12}+r / 2\right)|\hat{o}| \phi_{a_{2}}\left(q_{12}-r / 2\right)\right\rangle$ be represented by a Taylor expansion around $q_{1}=q_{2}=q_{12} \equiv\left(q_{1}+q_{2}\right) / 2$ up to second order.

In transport theories, this approximation is customarily called the "Fokker-Planck approximation (FPA)". It was introduced in order to replace the "master equation" which is an integro-differential equation for a classical probability distribution depending on slowly varying variables, by simple differential equations of second order, the so-called "Fokker-Planck equations (FPE)". A pertinent presentation of this procedure is given in Ref. [17].

$\underline{\text { Section } 5}$ of our paper will be devoted to the fluctuation-dissipation relation: It will be shown that, in the framework of our approach, such a relation holds for those transport coefficients in the NZ equation which are multiplied with the "diagonal" part $R\left(q_{1}, q_{1} ; t\right)$ of the reduced density matrix $R\left(q_{1}, q_{2} ; t\right)$ (see Eq. (5.10) $)$. Furthermore, as will become clear, the derivation of the fluctuation-dissipation relation relies on a few additional approximations beyond the mere perturbative treatment of the coupling Hamiltonian $\hat{H}_{c p l}$. 
As we take into account the occurrence of intrinsic excitations of the fissioning or fusing system, our theory may be applied up to moderately high excitation energies. The upper limit of its applicability will be given by the appearance of final channels with more than two final composite particles, in particular, the "incomplete fusion" channels. In these reactions, only a part of the incoming projectile nucleus fuses and, subsequently, undergoes fission, whereas the remaining part is immediately leaving the interaction zone in predominantly forward direction.

The emission of neutrons, protons, and photons by the fissioning nucleus will be described within the framework of our theory in a paper which we shall submit in due time to the same journal. For excitation energies in excess of about $50 \mathrm{MeV}$, the evaporation of neutrons and protons has been rather successfully described on the basis of the Thomas-Fermi approximation and a simple Fokker-Planck equation for the fissioning nucleus [18].

Whereas the intrinsic excitations can only be treated quantum-mechanically, the collective motion leading to fission via the passage through strongly deformed nuclear shapes can approximately be described as a classical motion, at least at excitation energies where tunnelling is negligible. Indeed, valuable insights into the fusion- and fissionprocesses have been obtained within classical models [19].

In our theory, the classical limit could be investigated by introducing the Wigner-Weyl representation for the statistical operator and by replacing commutators by Poisson brackets. We refrain from going into these details in the present paper.

In Section 6, we discuss the relation of our theory to earlier work on the microscopic theory of nuclear fission.

In Section 7, we summarize our results and point out open problems.

\section{Choice of basis functions}

The choice of the basis-functions is of great importance in our theory, because their dependence on the generator coordinates $q$ enters our description of the slow collective motion and of the repartition of the intrinsic excitation energy.In the two subsections of this chapter, we deal with the biorthogonal basis and with a physically motivated special choice of an orthonormal basis.

The Hill-Wheeler (HW) states introduced in Eq. (1.10) span a $\mathcal{N}$-dimensional vector space. If $\mathcal{N}_{g c}$ is the number of discrete values of the generator coordinates and if one associates a given number $\mathcal{N}_{\text {intr }}$ of intrinsic excitations with each given value of the generator coordinates, the dimension of the vector space is given by $\mathcal{N}=\mathcal{N}_{g c} \cdot \mathcal{N}_{\text {intr }}$.

The $\mathcal{N}$ Hill-Wheeler states $\phi_{a}(q)$ and their adjoints $\widetilde{\phi_{a}(q)}$ defined by the property 1.15 satisfy the completeness relation

$$
\sum_{a, q}\left|\phi_{a}(q)\right\rangle\left\langle\widetilde{\phi_{a}(q)}\left|=\sum_{a, q}\right| \widetilde{\phi_{a}(q)}\right\rangle\left\langle\phi_{a}(q)\right|=1
$$

Henceforth, we convene to use the ordinary HW-states as "ket"-states and the adjoints as "bra"-states.

The adjoint states are obtained from the ordinary HW-states through the inverse $\left(N^{-1}\right)_{a_{1} a_{2}}\left(q_{1}, q_{2}\right)$ of the overlap matrix (1.12), as can be easily seen :

$$
\begin{aligned}
& \left\langle\widehat{\phi_{a_{1}}\left(q_{1}\right)}\right|=\sum_{a_{2} q_{2}}\left\langle\widehat{\phi_{a_{1}}\left(q_{1}\right)} \mid \widetilde{\phi_{a_{2}}\left(q_{2}\right)}\right\rangle\left\langle\phi_{a_{2}}\left(q_{2}\right)\right| \\
& \left\langle\widetilde{\phi_{a_{1}}\left(q_{1}\right)} \mid \widehat{\phi_{a_{2}}\left(q_{2}\right)}\right\rangle=\left(N^{-1}\right)_{a_{1} a_{2}}\left(q_{1}, q_{2}\right)
\end{aligned}
$$

The labels $a_{1}, q_{1}$ characterizing a given adjoint state $\widetilde{\phi_{a_{1}}\left(q_{1}\right)}$ denote the state with the largest amplitude $\left(N^{-1}\right)_{a_{1} a_{2}}\left(q_{1}, q_{2}\right)$ in Eq. (2.2). We write the overlap matrix in the form

$$
N=1+K
$$

The eigenvalues of the matrix $K$ lie within the unit circle. Consequently, the series expansion for the inverse $N^{-1}$ converges

$$
N^{-1}=(1+K)^{-1}=1-K+K^{2}-K^{3}+\ldots
$$

The order of magnitude of the term $K^{n}$ in Eq. (2.5) is given by $\varepsilon^{n}$ where the "overlap parameter" $\varepsilon$ is defined by

$$
\varepsilon:=\exp \left(-\Delta q^{2} / 2 \sigma_{0}^{2}\right)
$$


The parameter $\Delta q$ is the distance between neighbouring discrete values of the generator coordinate $q$. To each type of generator coordinate corresponds a certain parameter $\varepsilon$.

In Fig.1, we present the overlap matrix $N$ for the simple case of 6 different HW-states.

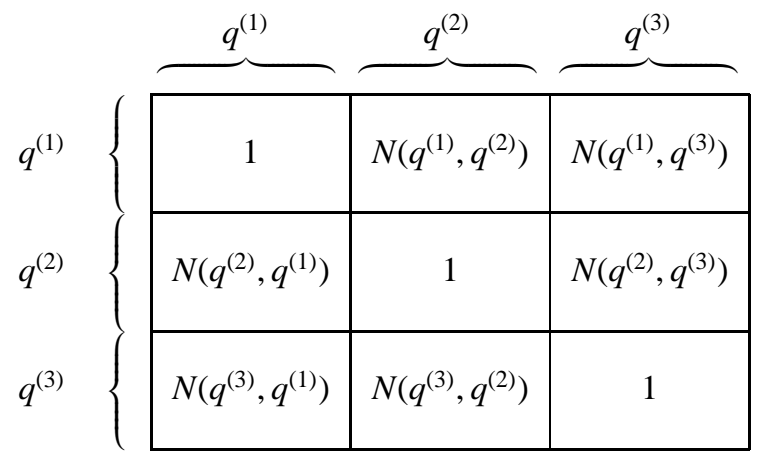

Figure 1: Form of the overlap matrix $N$ for the case of 3 values $\left(q^{(1)}, q^{(2)}, q^{(3)}\right)$ of the g.c. and 2 states of intrinsic motion $\left(a^{(1)}, a^{(2)}\right)$. The $2 \times 2$ submatrices for given values $q^{(i)} \neq q^{(j)}$ and $q^{(i)}=q^{(j)}$ of the g.c. are denoted by $N\left(q^{(i)}, q^{(j)}\right)$ and 1 , respectively.

The matrix-elements of a hermitian operator $\hat{O}=\hat{O}^{\dagger}$ between biorthogonal states satisfy the condition

$$
O_{a_{1} a_{2}}\left(q_{1}, q_{2}\right):=\left\langle\widehat{\phi_{a_{1}}\left(q_{1}\right)}|\hat{O}| \phi_{a_{2}}\left(q_{2}\right)\right\rangle=\left\langle\phi_{a_{2}}\left(q_{2}\right)|\hat{O}| \widetilde{\phi_{a_{1}}\left(q_{1}\right)}\right\rangle^{*}
$$

The matrix $O \equiv\left\{O_{a_{1} a_{2}}\left(q_{1}, q_{2}\right)\right\}$ is thus not hermitian. The lack of hermiticity of the biorthogonal matrix representation of a hermitian operator $\hat{O}$ is of no consequence for the thermodynamic mean value

$$
\langle\langle\hat{O}\rangle\rangle:=\operatorname{Tr}(\hat{\rho}(t) \hat{O})
$$

given the fact that the trace is independent of the representation in which it is evaluated.

Let us note that the adjoint basis-states $\widetilde{\phi_{a}(q)}$ have no physical meaning and that the operators

$$
\hat{\Pi}_{a}(q):=\left|\phi_{a}(q)\right\rangle\left\langle\widetilde{\phi_{a}(q)}\right|
$$

although satisfying the property

$$
\hat{\Pi}_{a}(q) \hat{\Pi}_{a^{\prime}}\left(q^{\prime}\right)=\delta_{a a^{\prime}} \delta_{q q^{\prime}} \hat{\Pi}_{a}(q)
$$

are not projection operators in the usual sense, as they are not hermitian.

One can avoid the inconveniences of the representation in terms of biorthogonal basis functions by introducing a complete system of orthonormal basis states in the $\mathcal{N}$-dimensional HW-space. There are, of course, infinitely many possible choices of orthonormal basis functions. One possibility would be to use the orthonormal eigenstates of the overlap matrix $N$ as a system of basis functions.

A more physical choice is to determine the eigenstates of the hermitian operator

$$
\hat{H}^{M}:=\sum_{a_{1} q_{1} a_{2} q_{2}}\left|\widetilde{\phi_{a_{1}}\left(q_{1}\right)}\right\rangle \mathcal{H}_{a_{1} a_{2}}^{M}\left(q_{1}, q_{2}\right)\left\langle\widetilde{\phi_{a_{2}}\left(q_{2}\right)}\right|
$$

where the hermitian matrix $\mathcal{H}_{a_{1} a_{2}}^{M}\left(q_{1}, q_{2}\right)$ is defined by

$$
\mathcal{H}_{a_{1} a_{2}}^{M}\left(q_{1}, q_{2}\right):=\frac{1}{2}\left(E_{1 a_{1}}^{\prime}\left(q_{1}\right)+E_{1 a_{2}}^{\prime}\left(q_{2}\right)\right) N_{a_{1} a_{2}}\left(q_{1}, q_{2}\right)
$$

The energies $E_{1 a}^{\prime}(q)$ pertain to states of independently moving quasi-particles in a nucleus whose shape is defined by the g.c. $q$ (see Eq. (1.10)). The orthonormal eigenstates $\Psi_{\alpha}$ of the Hamiltonian (2.11) with eigenvalues $\varepsilon_{\alpha}$

$$
\left\langle\Psi_{\beta}\left|\hat{H}^{M}\right| \Psi_{\alpha}\right\rangle=\varepsilon_{\alpha}\left\langle\Psi_{\beta} \mid \Psi_{\alpha}\right\rangle=\varepsilon_{\alpha} \delta_{\alpha \beta}
$$


can be expanded in terms of the HW-states $\phi_{a}(q)$ or their adjoints

$$
\left|\Psi_{\alpha}\right\rangle=\sum_{a^{\prime}, q^{\prime}}\left|\phi_{a^{\prime}}\left(q^{\prime}\right)\right\rangle\left\langle\widehat{\phi_{a^{\prime}}\left(q^{\prime}\right)} \mid \Psi_{\alpha}\right\rangle=\sum_{a^{\prime}, q^{\prime}}\left|\widetilde{\phi_{a^{\prime}}\left(q^{\prime}\right)}\right\rangle\left\langle\phi_{a^{\prime}}\left(q^{\prime}\right) \mid \Psi_{\alpha}\right\rangle
$$

In the limit of vanishing overlap parameter $\varepsilon$, a given eigenstate $\Psi_{\alpha}$ becomes equal to a specific HW-state $\phi_{a}(q)$. Thus, we can denote the orthonormal basis states $\Psi_{\alpha}$ by $\Psi_{a}(q)$ where the labels $a, q$ characterize the main component $\phi_{a}(q)$ in Eq. (2.14) which survives for $\varepsilon \rightarrow 0$.

The coefficients

$$
\begin{aligned}
D_{a q}^{\beta} & :=\left\langle\phi_{a}(q) \mid \Psi_{\beta}\right\rangle \\
\widetilde{D}_{\alpha}^{a q} & :=\left\langle\widetilde{\phi_{a}(q)} \mid \Psi_{\alpha}\right\rangle
\end{aligned}
$$

satisfy the relations

$$
\begin{aligned}
& \sum_{a q} D_{a q}^{\beta^{*}} \widetilde{D}_{\alpha}^{a q}=\left\langle\Psi_{\beta} \mid \Psi_{\alpha}\right\rangle=\delta_{\alpha \beta} \\
& \sum_{\alpha} \widetilde{D}_{\alpha}^{a_{2} q_{2}} D_{a_{1} q_{1}}^{\alpha^{*}}=\delta_{a_{1} a_{2}} \delta_{q_{1} q_{1}} \\
& D_{a_{1} q_{1}}^{\beta}=\left\langle\phi_{a_{1}}\left(q_{1}\right) \mid \Psi_{\beta}\right\rangle=\sum_{a_{2} q_{2}} N_{a_{1} a_{2}}\left(q_{1}, q_{2}\right) \widetilde{D}_{\beta}^{a_{2} q_{2}} \\
& \widetilde{D}_{\beta}^{a_{1} q_{1}}=\left\langle\widehat{\phi_{a_{1}}\left(q_{1}\right)} \mid \Psi_{\beta}\right\rangle=\sum_{a_{2} q_{2}}\left(N^{-1}\right)_{a_{1} a_{2}}\left(q_{1}, q_{2}\right) D_{a_{2} q_{2}}^{\beta}
\end{aligned}
$$

Eq. (2.13) can be written in the form

$$
\sum_{a_{1} q_{1} a_{2} q_{2}} \widetilde{D}_{\beta}^{a_{1} q_{1}{ }^{*}} \mathcal{H}_{a_{1} a_{2}}^{M}\left(q_{1}, q_{2}\right) \widetilde{D}_{\alpha}^{a_{2} q_{2}}=\varepsilon_{\alpha} \delta_{\alpha \beta}
$$

and the eigenenergies $\varepsilon_{\alpha}$ can also be obtained as solutions of the equation

$$
\operatorname{det}\left\{\mathcal{H}_{a_{1} a_{2}}^{M}\left(q_{1}, q_{2}\right)-\varepsilon_{\alpha} N_{a_{1} a_{2}}\left(q_{1}, q_{2}\right)\right\}=0
$$

In the case that the overlap parameter $\varepsilon$ is noticeably smaller than 1 , a given eigenstate $\Psi_{\alpha}=\Psi_{a}(q)$ of $\hat{H}^{M}$ describes physically a state of independent quasi-particles whose main component is the eigenstate $\phi_{a}(q)$ of the HB-Hamiltonian $\hat{H}_{1}^{\prime}(q)$. However, it contains admixtures of eigenstates $\phi_{a^{\prime}}\left(q^{\prime}\right)$ of the HB-Hamiltonian $\hat{H}_{1}^{\prime}\left(q^{\prime}\right)$ with $q^{\prime}=q \pm \Delta q, q \pm$ $2 \Delta q, \ldots$

Let us note that the operator defined by

$$
\hat{\Pi}_{\alpha}=\left|\Psi_{\alpha}\right\rangle\left\langle\Psi_{\alpha}|=| \Psi_{a}(q)\right\rangle\left\langle\Psi_{a}(q)\right|
$$

is a true projection operator satisfying not only the relations

$$
\hat{\Pi}_{\alpha} \hat{\Pi}_{\beta}=\delta_{\alpha \beta} \hat{\Pi}_{\alpha}
$$

but also

$$
\hat{\Pi}_{\alpha}=\hat{\Pi}_{\alpha}^{\dagger}
$$

As most of the results to be obtained in the following chapters hold in the same form for the matrix-representation in terms of the biorthogonal or the orthonormal basis-functions, we introduce a unified notation encompassing the two alternatives in the following table 


\begin{tabular}{|c|c|c|}
\hline $\begin{array}{c}\text { unified } \\
\text { notation }\end{array}$ & $\begin{array}{c}\text { biorthogonal } \\
\text { basis }\end{array}$ & $\begin{array}{c}\text { orthonormal } \\
\text { basis }\end{array}$ \\
\hline$|a q\rangle$ & $\left|\phi_{a}(q)\right\rangle$ & $\left|\Psi_{a}(q)\right\rangle$ \\
\hline$\langle a q|$ & $\left\langle\widetilde{\phi_{a}(q)}\right|$ & $\left\langle\Psi_{a}(q)\right|$ \\
\hline $\mathcal{E}_{a}^{\prime}(q)$ & $E_{1 a}^{\prime}(q)$ & $\varepsilon_{\alpha}^{\prime}(q)$ \\
\hline$\left\langle a_{1} q_{1}|\hat{O}| a_{2} q_{2}\right\rangle$ & $\left\langle\widehat{\left.\phi_{a_{1}}\left(q_{1}\right)|\hat{O}| \phi_{a_{2}}\left(q_{2}\right)\right\rangle}\right.$ & $\left\langle\Psi_{a_{1}}\left(q_{1}\right)|\hat{O}| \Psi_{a_{2}}\left(q_{2}\right)\right\rangle$ \\
\hline
\end{tabular}

\section{Derivation of an equation of motion for the slowly time-dependent density operator $\hat{\rho}_{P}(t)$ and introduction of perturbation- and Markov- approximations}

The theory to be presented in this paper is devised to describe the slowly time-dependent average evolution of a fission- or fusion-process at moderate excitation energies. It should thus be applicable to reactions which proceed via the formation of compound nuclear resonances ("CNR") with a lifetime which is much larger than the time scale of its formation $\left(\lesssim 10^{-22} \mathrm{~s}\right)$. The CNR are highly complicated nuclear many-body states which exhibit a finite width due to their coupling to the continua of the open decay channels. It is impossible to determine the detailed form of these states. According to the deep original insight of N. Bohr [20], the detailed structure of the CNRs is not important for the understanding of the slow time-evolution of the system in the fission channel which is the topic of the present paper. In the language of transport theory, Bohr's hypothesis implies that the "memory time" of the system is much smaller than the time-scale of the collective motion in the fission channel. Consequently, we may describe the slow collective time-evolution of the fission process starting from a simple initial condition for the collective flow. We shall come back to this question when discussing the "transport equation" we are going to derive.

One usually considers the decay of an ensemble of $\mathrm{CN}$ resonances. But even in the case that we study the decay of a single, isolated CN resonance, the system should be described by the "density operator" (="statistical operator") $\hat{\rho}(t)$ rather than by a wavefunction. The reason is that it is the density operator which expresses the information we have on a physical system and it is this quantity which simplifies when we ask for a reduced information only.

The statistical operator satisfies the von Neumann-Liouville equation

$$
\dot{\hat{\rho}}(t)=\frac{-i}{\hbar}[\hat{H}, \hat{\rho}(t)]
$$

In our case, $\hat{H}$ is the Hamiltonian of $A$ nucleons ( $Z$ protons and $N$ neutrons) interacting by effective nucleon-nucleon interactions $v$ :

$$
\hat{H}=-\sum_{i=1}^{A} \frac{\hbar^{2}}{2 M} \Delta(i)+\frac{1}{2} \sum_{i, j=1}^{A} v\left(x_{i}, x_{j}\right)
$$

The symbol $x_{i}$ denotes the position, spin and isospin variables of the $i$-th nucleon and $M$ is the mass of the nucleon. We emphasize that the knowledge of the nucleon-nucleon interaction represents the only nuclear input information in our theory.

The statistical operator $\hat{\rho}(t)$ describes the entire time evolution, i.e. the slow collective change of the nucleonic distribution during fission as well as the rapidly changing state of internal motion which involves a superposition of many complicated intrinsic excitation modes. Of course, we also need a knowledge of the initial state $\hat{\rho}(0)$. Due to the effect of memory loss mentioned above, it is probably innocuous that we only have a rather incomplete information on $\hat{\rho}(0)$.

At the origin of any transport theory, one has to introduce the part $\hat{\rho}_{P}(t)$ of the total density operator which should describe the slowly proceeding dynamical evolution of the system only. As we do not have independent collective degrees of freedom at our disposal, we have to define the density operator $\hat{\rho}_{P}(t)$ in terms of an "ansatz" for the matrixelements $\left\langle a_{1} q_{1}\left|\hat{\rho}_{P}(t)\right| a_{2} q_{2}\right\rangle$ of $\hat{\rho}_{P}(t)$ with respect to the basis-states introduced in Section 2.

As the slow collective time change is not expected to depend on the precise form of the intrinsic motion, let us first define a "reduced density matrix"

$$
R\left(q_{1}, q_{2} ; t\right) \equiv \rho^{r e d}\left(q_{1}, q_{2} ; t\right):=\sum_{a}\left\langle a q_{1}|\hat{\rho}(t)| a q_{2}\right\rangle
$$


as the part of the operator $\hat{\rho}_{P}(t)$ which is to describe the dynamics of the shape changes and, thereafter, of the motion of the fission fragments in space.

Furthermore, we introduce a grand canonical distribution of the system with regard to the eigen-energies $\mathcal{E}_{a}(q)$ of the basis states

$$
F_{a}(q, T(q, t)):=\frac{1}{Z_{0}(q, T)} \exp \left(-\beta(q, t) \mathcal{E}_{a}^{\prime}(q)\right)
$$

Here, $\beta$ is the reciprocal temperature

$$
\beta(q, T)=\frac{1}{T(q, t)}
$$

and $Z_{0}(q, T)$ the partition function

$$
Z_{0}(q, T):=\sum_{a} e^{-\beta(q, t) \mathcal{E}_{a}^{\prime}(q)}
$$

The temperature $T$ may depend on the g.c. $q$ and on the time $t$. The choice of the temperature $T(q, t)$ as a function of $q$ and $t$ will be presented in Section 4. Let us only note that, for the case of a "nuclear transport theory", the "temperature" is not determined externally by the contact of the system with a bath, i.e. a large reservoir at a given temperature. Rather, we must consider it as a parameter which we have to choose so as to optimize the "ansatz" $\hat{\rho}_{P}(t)$ for the slowly time-dependent part of the density operator $\hat{\rho}(t)$.

We define the matrix representation of $\hat{\rho}_{P}(t)$ by

$$
\left\langle a_{1} q_{1}\left|\hat{\rho}_{P}(t)\right| a_{2} q_{2}\right\rangle:=\delta_{a_{1} a_{2}} R\left(q_{1}, q_{2} ; t\right) F_{a_{2}}\left(q_{2}, T\left(q_{2}, t\right)\right)
$$

The Boltzmann distribution (3.4) which appears in the ansatz (3.7) is customarily also written in the form

$$
F_{a}(q, T(q, t))=\exp \left[-\beta(q, t)\left(\mathcal{E}_{a}^{\prime}(q)-G(q, T)\right)\right]
$$

where the Gibbs function $G(q, T)$ is related to the partition function (3.6) by

$$
G(q, T)=-T(q, t) \ln Z_{0}(q, T)
$$

The following idea underlies the choice of the canonical distribution of the system with respect to the intrinsic excitations in Eq. (3.7):

As $\hat{\rho}_{P}(t)$ should only describe the slow time-evolution of the system, the precise state of the intrinsic motion is not believed to be relevant. The choice of the canonical distribution as approximation may be motivated by the observation that the distribution corresponds to the minimal information (=maximal entropy) on the state of internal motion (see Section 4).

We draw attention to the fact that the matrix (3.7) does not define a hermitian operator $\hat{\rho}_{P}(t)$, even if we use orthonormal basis states $|a q\rangle$. In addition, let us note that the reduced matrix $R\left(q_{1}, q_{2} ; t\right)$ is hermitian only if, in Eq. (3.3), we use an orthonormal basis. It is not hermitian if we use the biorthogonal basis. A further discussion of the question of hermiticity will be given in the Appendix $\mathrm{C}$

The non-hermitian character of $\hat{\rho}_{P}(t)$ is of no consequence as far as mean-values of observables $\hat{O}=\hat{O}^{\dagger}$ are concerned. We just have to evaluate the mean values with the hermitian part of $\hat{\rho}_{P}(t)$ which is defined by

$$
\hat{\rho}_{P}^{h}(t)=\frac{1}{2}\left(\hat{\rho}_{P}(t)+\hat{\rho}_{P}^{\dagger}(t)\right)
$$

We find

$$
\begin{aligned}
\langle\langle\hat{O}\rangle\rangle & :=\operatorname{Tr}\left(\hat{\rho}_{P}^{h}(t) \hat{O}\right)=\sum_{a_{1} q_{1} a_{2} q_{2}} \operatorname{Re}\left\{\left\langle a_{1} q_{1}\left|\hat{\rho}_{P}(t)\right| a_{2} q_{2}\right\rangle\left\langle a_{2} q_{2}|\hat{O}| a_{1} q_{1}\right\rangle\right\} \\
& =\operatorname{Re}\left\{\operatorname{Tr}\left(\hat{\rho}_{P}(t) \hat{O}\right)\right\}
\end{aligned}
$$

We also note that, for orthonormal basis states $\Psi_{a}(q)$, we can interpret

$$
R(q, q ; t):=\sum_{a}\left\langle\Psi_{a}(q)|\hat{\rho}(t)| \Psi_{a}(q)\right\rangle
$$


as the probability to find the system in any one of the basis states $\Psi_{a}(q)$ for given $q$. In the case of the biorthogonal basis, the corresponding quantity

$$
R(q, q ; t):=\sum_{a}\left\langle\widetilde{\phi_{a}(q)}|\hat{\rho}(t)| \phi_{a}(q)\right\rangle
$$

cannot be interpreted as a probability, as $\left|\widetilde{\phi_{a}(q)}\right\rangle\left\langle\phi_{a}(q)\right|$ is not a hermitian projection operator contrary to $\left|\Psi_{a}(q)\right\rangle\left\langle\Psi_{a}(q)\right|$.

We can write the operator $\hat{\rho}_{P}(t)$ as a projection operator $\hat{P}$ acting on $\hat{\rho}(t)$ :

$$
\hat{\rho}_{P}(t)=\hat{P} \hat{\rho}(t)
$$

Writing $\left\langle a_{1} q_{1}\left|\hat{\rho}_{P}(t)\right| a_{2} q_{2}\right\rangle$ as defined by Eq. (3.7) in the form

$$
\left\langle a_{1} q_{1}|\hat{P} \hat{\rho}(t)| a_{2} q_{2}\right\rangle=\sum_{a_{3} q_{3} a_{4} q_{4}} P_{a_{1} q_{1} a_{2} q_{2} a_{3} q_{3} a_{4} q_{4}}\left\langle a_{3} q_{3}|\hat{\rho}(t)| a_{4} q_{4}\right\rangle
$$

one finds that the matrix representation of the projection operator $\hat{P}$ has the form

$$
P_{a_{1} q_{1} a_{2} q_{2} a_{3} q_{3} a_{4} q_{4}}=\delta_{a_{1} a_{2}} \delta_{q_{1} q_{3}} \delta_{q_{2} q_{4}} \delta_{a_{3} a_{4}} F_{a_{2}}\left(q_{2}, T\left(q_{2}, t\right)\right)
$$

Using the definition (3.16) of $\hat{P}$ we define a complementary projection operator

$$
\hat{Q}=1-\hat{P}
$$

by the matrix representation

$$
Q_{a_{1} q_{1} a_{2} q_{2} a_{3} q_{3} a_{4} q_{4}}=\delta_{a_{1} a_{3}} \delta_{q_{1} q_{3}} \delta_{a_{2} a_{4}} \delta_{q_{2} q_{4}}-P_{a_{1} q_{1} a_{2} q_{2} a_{3} q_{3} a_{4} q_{4}}
$$

One easily checks that the following relations hold:

$$
\hat{P}^{2}=\hat{P} ; \quad \hat{Q}^{2}=\hat{Q} ; \quad \hat{P} \hat{Q}=\hat{Q} \hat{P}=0
$$

Through their dependence on the (slowly) time-dependent temperature, the projection operators are slowly timedependent quantities. The total density operator $\hat{\rho}(t)$ can be decomposed into the slowly time-dependent part $\hat{\rho}_{P}(t)$ and the remainder $\hat{\rho}_{Q}(t)$ :

$$
\hat{\rho}(t)=(\hat{P}+\hat{Q}) \hat{\rho}(t)=\hat{\rho}_{P}(t)+\hat{\rho}_{Q}(t)
$$

The part $\hat{\rho}_{Q}(t)$ of the statistical operator describes rapidly time-dependent processes and involves summations over a large number of terms with different phases. Whereas the detailed form of $\hat{\rho}_{Q}(t)$ will be considered in the next Section, let us note the properties

$$
\begin{aligned}
& \sum_{a}\left\langle a q_{1}\left|\hat{\rho}_{Q}(t)\right| a q_{2}\right\rangle=0 \\
& \operatorname{Tr}\left(\hat{\rho}_{Q}(t) \hat{O}^{r e d}\right)=0
\end{aligned}
$$

Here, $\hat{O}^{\text {red }}$ is an operator the matrix-representation of which is diagonal with respect to the quantum numbers of the intrinsic motion:

$$
\left\langle a_{2} q_{2}\left|\hat{O}^{r e d}\right| a_{1} q_{1}\right\rangle:=\delta_{a_{2} a_{1}} O\left(q_{2}, q_{1}\right)
$$

Obviously, observables with the property (3.22) put to the test purely collective features of the system. Examples are the charge- and mass- numbers and the kinetic energy of relative motion of the fission fragments.

For deriving an equation of motion for the operator $\hat{\rho}_{P}(t)$, we make use of the formalism introduced by Nakajima [14] and Zwanzig [15]. Acting on the Eq. 3.1] with the projection operators $\hat{P}$ and $\hat{Q}$ and taking into account their time-dependence, we obtain the coupled equations

$$
\begin{aligned}
& \frac{d \hat{\rho}_{P}(t)}{d t}=-\frac{i}{\hbar} \hat{P} \hat{L}\left(\hat{\rho}_{P}(t)+\hat{\rho}_{Q}(t)\right)+\dot{\hat{P}}(t) \hat{\rho}(t) \\
& \frac{d \hat{\rho}_{Q}(t)}{d t}=-\frac{i}{\hbar} \hat{Q} \hat{L}\left(\hat{\rho}_{P}(t)+\hat{\rho}_{Q}(t)\right)+\dot{\hat{Q}}(t) \hat{\rho}(t)
\end{aligned}
$$


where the Liouvillean operator $\hat{L}$ acting on an operator $\hat{A}$ is defined by

$$
\hat{L} \hat{A}:=[\hat{H}, \hat{A}]
$$

Using some properties of projection operators and their time derivatives, which are derived and presented in Appendix A, we may write

$$
\dot{\hat{P}}(t) \hat{\rho}(t)=\dot{\hat{P}}(t)(\hat{P}(t)+\hat{Q}(t)) \hat{\rho}(t)=\dot{\hat{P}}(t) \hat{\rho}_{P}(t)
$$

and

$$
\dot{\hat{Q}}(t) \hat{\rho}(t)=-\dot{\hat{P}}(t) \hat{\rho}(t)=-\dot{\hat{P}}(t) \hat{\rho}_{P}(t)
$$

The coupled equations $(3.24)-(3.25)$ thus take the form

$$
\begin{aligned}
\frac{d \hat{\rho}_{P}}{d t} & =-\frac{i}{\hbar} \hat{P} \hat{L}\left(\hat{\rho}_{P}(t)+\hat{\rho}_{Q}(t)\right)+\dot{\hat{P}}(t) \hat{\rho}_{P}(t) \\
\frac{d \hat{\rho}_{Q}}{d t} & =-\frac{i}{\hbar} \hat{Q} \hat{L} \hat{\rho}_{Q}(t)+\hat{\sigma}(t)
\end{aligned}
$$

where the "source term" $\hat{\sigma}(t)$ is defined by

$$
\hat{\sigma}(t):=-\frac{i}{\hbar} \hat{Q}(t) \hat{L} \hat{\rho}_{P}(t)-\dot{\hat{P}}(t) \hat{\rho}_{P}(t)
$$

In order to obtain an equation of motion for the density operator $\hat{\rho}_{P}(t)$, we have to find a formal solution of the Eq. (3.30) and substitute it into Eq. (3.29). Proceeding in analogy to Ref. [21], we introduce a Green function $\hat{G}(t, s)$ as a solution of the equation

$$
\frac{\partial \hat{G}(t, s)}{\partial t}+\frac{i}{\hbar} \hat{Q}(t) \hat{L} \hat{G}(t, s)=\hat{Q}(t) \delta(t-s)
$$

and write the operator $\hat{\rho}_{Q}(t)$ for $t>0$ in the form

$$
\hat{\rho}_{Q}(t)=\hat{G}(t, 0) \hat{\rho}_{Q}(0)+\int_{0}^{\infty} d s \hat{G}(t, s) \hat{Q}(s) \hat{\sigma}(s)
$$

In the Appendix $\mathrm{A}$ it is shown that the operator $\dot{\hat{P}}(t)$ has the property

$$
\hat{P}(t) \dot{\hat{P}}(t)=0
$$

which implies

$$
\dot{\hat{P}}(t)=(\hat{P}(t)+\hat{Q}(t)) \dot{\hat{P}}(t)=\hat{Q}(t) \dot{\hat{P}}(t)
$$

Consequently, the source term $\hat{\sigma}(s)$ satisfies

$$
\hat{Q}(s) \hat{\sigma}(s)=\hat{\sigma}(s)
$$

and we may write, instead of Eq. (3.33), more simply

$$
\hat{\rho}_{Q}(t)=\hat{G}(t, 0) \hat{\rho}_{Q}(0)+\int_{0}^{\infty} d s \hat{G}(t, s) \hat{\sigma}(s)
$$

It is easily checked that the ansatz (3.37) together with the defining equation (3.32) for the Green operator $\hat{G}(t, s)$ represents the solution of the Eq. (3.30) with the initial value $\hat{\rho}_{Q}(0)$ :

$$
\begin{aligned}
& \frac{d \hat{\rho}_{Q}(t)}{d t} \stackrel{3.37}{=} \frac{\partial \hat{G}(t, 0)}{\partial t} \hat{\rho}_{Q}(0)+\int_{0}^{\infty} d s \frac{\partial \hat{G}(t, s)}{\partial t} \hat{\sigma}(s) \\
& \stackrel{3.32}{=}\left[-\frac{i}{\hbar} \hat{Q}(t) \hat{L} \hat{G}(t, 0)+\hat{Q}(t) \delta(t)\right] \hat{\rho}_{Q}(0)+\int_{0}^{\infty} d s\left[-\frac{i}{\hbar} \hat{Q}(t) \hat{L} \hat{G}(t, s)+\hat{Q}(t) \delta(t-s)\right] \hat{\sigma}(s) \\
& \frac{d \hat{\rho}_{Q}(t)}{d t} \stackrel{3.37}{=}-\frac{i}{\hbar} \hat{Q}(t) \hat{L} \hat{G}(t, 0) \hat{\rho}_{Q}(0)+\hat{Q}(0) \hat{\rho}_{Q}(0) \delta(t)-\frac{i}{\hbar} \hat{Q}(t) \hat{L}\left[\hat{\rho}_{Q}(t)-\hat{G}(t, 0) \hat{\rho}_{Q}(0)\right]+\theta_{0}(t) \hat{Q}(t) \hat{\sigma}(t)
\end{aligned}
$$


Apart from the term $\hat{Q}(0) \hat{\rho}_{Q}(0) \delta(t)$, which expresses the initial condition for $\hat{\rho}_{Q}(t)$, the r.h.s. of Eq. (3.38) is seen to agree for $t>0$ with the r.h.s. of Eq. (3.30).

Substituting the formal solution (3.37) into the Eq. 3.29, we obtain the desired integro-differential equation for $\hat{\rho}_{P}(t)$ :

$$
\begin{aligned}
\frac{d \hat{\rho}_{P}(t)}{d t}= & -\frac{i}{\hbar} \hat{P}(t) \hat{L} \hat{G}(t, 0) \hat{\rho}_{Q}(0)-\frac{i}{\hbar} \hat{P}(t) \hat{L} \hat{\rho}_{P}(t) \\
& -\frac{i}{\hbar} \hat{P}(t) \hat{L} \int_{0}^{\infty} d s \hat{G}(t, s) \hat{\sigma}(s)+\hat{\hat{P}}(t) \hat{\rho}_{P}(t)
\end{aligned}
$$

We will refer to this equation as "Nakajima-Zwanzig (NZ)"-equation. It is a rigorously valid equation of motion for the slowly time-dependent part $\hat{\rho}_{P}(t)$ of the total density operator $\hat{\rho}(t)$.

The formal solution of the equation (3.32) for the Green operator $\hat{G}(t, s)$ can be written as the following timeordered product

$$
\hat{G}(t, s)=\theta_{0}(t-s) \hat{Q}(t) \hat{T}\left\{e^{-\int_{s}^{t} d \tau\left[\frac{i}{\hbar} \hat{L} \hat{Q}(\tau)-\dot{\hat{P}}(\tau)\right]}\right\}
$$

$\hat{T}$ denoting the time-ordering operator. The detailed proof that 3.40 is a solution of Eq. 3.32 is given in the Appendix B Through its dependence on the Green operator $\hat{G}(t, s)$, the NZ-equation (3.39) still contains the full complexity of the total system. Nevertheless, this equation has the merit to display the different physical processes which contribute to the time evolution of $\hat{\rho}_{P}(t)$.

In order to describe the physical meaning of the different terms in the equation of motion (3.39), it is useful to decompose the matrix $\left\langle a_{1} q_{1}|\hat{A}| a_{2} q_{2}\right\rangle$ representing an arbitrary operator $\hat{A}$ into two parts using the projection operators $\hat{P}$ and $\hat{Q}$ :

$$
\begin{aligned}
& \left\langle a_{1} q_{1}|\hat{A}| a_{2} q_{2}\right\rangle=\left\langle a_{1} q_{1}|\hat{P} \hat{A}| a_{2} q_{2}\right\rangle+\left\langle a_{1} q_{1}|\hat{Q} \hat{A}| a_{2} q_{2}\right\rangle \\
& \left\langle a_{1} q_{1}|\hat{P} \hat{A}| a_{2} q_{2}\right\rangle=\delta_{a_{1} a_{2}} F_{a_{2}}\left(q_{2}, T\left(q_{2}, t\right)\right) A^{r e d}\left(q_{1}, q_{2}\right) \\
& A^{r e d}\left(q_{1}, q_{2}\right):=\sum_{a}\left\langle a q_{1}|\hat{A}| a q_{2}\right\rangle \\
& \left\langle a_{1} q_{1}|\hat{Q} \hat{A}| a_{2} q_{2}\right\rangle:=\left\langle a_{1} q_{1}|\hat{A}| a_{2} q_{2}\right\rangle-\delta_{a_{1} a_{2}} F_{a_{2}}\left(q_{2}, T\left(q_{2}, t\right)\right) A^{r e d}\left(q_{1}, q_{2}\right)
\end{aligned}
$$

We shall refer to the two parts $\left\langle a_{1} q_{1}|\hat{P} \hat{A}| a_{2} q_{2}\right\rangle$ and $\left\langle a_{1} q_{1}|\hat{Q} \hat{A}| a_{2} q_{2}\right\rangle$ as the "canonical" or "P-part" and the "noncanonical" or " $Q$-part" of the matrix $\left\langle a_{1} q_{1}|\hat{A}| a_{2} q_{2}\right\rangle$.

Obviously, the Eq. (3.41) generalizes the decomposition of the density operator $\hat{\rho}$ to one of an arbitrary operator $\hat{A}$. Whereas $\left\langle a_{1} q_{1}|\hat{P} \hat{A}| a_{2} q_{2}\right\rangle$ depends on intrinsic excitations only through a canonical distribution, the complementary part $\left\langle a_{1} q_{1}|\hat{Q} \hat{A}| a_{2} q_{2}\right\rangle$ is expected to depend on the quantum numbers $a_{1}$ and $a_{2}$ in a complicated, possibly almost random way.

The nature of the different terms in the NZ-equation (3.39) can be described as follows:

Except for the term $\dot{\hat{P}}(t) \hat{\rho}_{P}(t)$, all terms on the r.h.s. of the NZ-equation 3.39) contain the projection operator $\hat{P}(t)$ on their left, which means that their matrix representation is of the form $\underline{3.42}$, (3.43). The term $\dot{\hat{P}}(t) \hat{\rho}_{P}(t)$ has the matrix representation

$$
\begin{aligned}
\left\langle a_{1} q_{1}\left|\dot{\hat{P}}(t) \hat{\rho}_{P}(t)\right| a_{2} q_{2}\right\rangle & =\sum_{a_{3} q_{3} a_{4} q_{4}} \dot{P}_{a_{1} q_{1} a_{2} q_{2} a_{3} q_{3} a_{4} q_{4}}\left\langle a_{3} q_{3}\left|\hat{\rho}_{P}(t)\right| a_{4} q_{4}\right\rangle \\
& =\delta_{a_{1} a_{2}} \frac{\partial F_{a_{2}}\left(q_{2}, T\left(q_{2}, t\right)\right)}{\partial T} \dot{T}\left(q_{2}, t\right) R\left(q_{1}, q_{2} ; t\right)
\end{aligned}
$$

where the derivative $\partial F_{a_{2}} / \partial T$ can be written in the form

$$
\frac{\partial F_{a_{2}}\left(q_{2}, T\left(q_{2}, t\right)\right)}{\partial T}=\frac{F_{a_{2}}\left(q_{2}, T\left(q_{2}, t\right)\right)}{T}\left\{\frac{\mathcal{E}_{a_{2}}^{\prime}\left(q_{2}\right)-G\left(q_{2}, T\right)}{T}+\frac{\partial G\left(q_{2}, T\right)}{\partial T}\right\}
$$


Introducing the entropy $S\left(q_{2}, T\right)$ of the system at the given value $q_{2}$ of the g.c.

$$
S\left(q_{2}, T\right)=-\frac{\partial G\left(q_{2}, T\right)}{\partial T}
$$

we can present the term $\left\langle a_{1} q_{1}\left|\dot{\hat{P}}(t) \hat{\rho}_{P}(t)\right| a_{2} q_{2}\right\rangle$ as follows

$$
\left\langle a_{1} q_{1}\left|\dot{\hat{P}}(t) \hat{\rho}_{P}(t)\right| a_{2} q_{2}\right\rangle=\delta_{a_{1} a_{2}} R\left(q_{1}, q_{2} ; t\right) F_{a_{2}}\left(q_{2}, T\left(q_{2}, t\right)\right) \frac{\dot{T}\left(q_{1}, t\right)}{T\left(q_{2}, t\right)}\left\{\frac{\mathcal{E}_{a_{2}}^{\prime}\left(q_{2}\right)-G\left(q_{2}, T\right)}{T}-S\left(q_{2}, T\left(q_{2}, t\right)\right)\right\}
$$

As the temperature is expected to be a slow function of time, the term is likely to be smaller than the other terms on the r.h.s of the NZ-equation. The role of the entropy will be discussed in Section 4

The inhomogeneous term $-i / \hbar \hat{P}(t) \hat{L} \hat{G}(t, 0) \hat{\rho}_{Q}(0)$ represents the memory of the system of the "non-canonical" part $\hat{\rho}_{Q}(0)$ of the initial density operator $\hat{\rho}(0)$. The operator $\hat{G}(t, 0)$ propagates $\hat{\rho}(0)$ from time 0 to time $t$, so that $-i / \hbar \hat{L} \hat{G}(t, 0) \hat{\rho}_{Q}(0)$ essentially represents the time-derivative of $\hat{G}(t, 0) \hat{\rho}_{Q}(0)$. Finally, the operator $\hat{P}(t)$ projects from it the component which is of $P$-type. One expects that the term $-i / \hbar \hat{P}(t) \hat{L} \hat{G}(t, 0) \hat{\rho}_{Q}(0)$ fades away after a short "memory time" $\tau_{\text {mem }} \leq 10^{-22} \mathrm{~s}$. In fact, the projection operator $\hat{P}$ filters from $\left\langle a_{1} q_{1}\left|\hat{L} \hat{G}(t, 0) \hat{\rho}_{Q}(0)\right| a_{2} q_{2}\right\rangle$ the "reduced part"

$$
\left\langle a_{1} q_{1}\left|\hat{P}(t) \hat{L} \hat{G}(t, 0) \hat{\rho}_{Q}(0)\right| a_{2} q_{1}\right\rangle=\delta_{a_{1} a_{2}} F_{a_{2}}\left(q_{2}, T\right) \sum_{a}\left\langle a q_{1}\left|\hat{L} \hat{G}(t, 0) \hat{\rho}_{Q}(0)\right| a q_{2}\right\rangle
$$

As the density operator $\hat{\rho}_{Q}(0)$ represents the complicated part of the initial density $\hat{\rho}(0)$ and as the propagator $\hat{G}(t, 0)$ tends to complicate further this part of the statistical operator, the $\hat{P}$-projection (3.49) is expected to become rapidly negligible.

In all what follows, the inhomogeneous term $-i / \hbar \hat{P}(t) \hat{L} \hat{G}(t, 0) \hat{\rho}_{Q}(0)$ of the NZ-equation will be neglected. The term was also neglected in all actual calculations of the fission dynamics we know of [4, 9, 10, 18].

Let us note that neglecting this term means essentially that one chooses the initial condition at a time where the collective motion towards fission has already started. The original compound system consists, at low excitation energy $(\lesssim 8 \mathrm{MeV})$, of several narrow $(\simeq 1 \mathrm{eV})$ compound nuclear resonances and, at high excitation energy $(\gtrsim 10 \mathrm{MeV})$, of overlapping resonances of an average width of $\gtrsim 100 \mathrm{keV}$. Thus, just after its formation, the compound system does not at all resemble a canonical distribution over intrinsic excitations as required by $\hat{\rho}_{P}(t)$, but rather a microcanonical ensemble of highly complex nuclear decaying states. The dynamical evolution of this initial state into one whose slowly time-dependent part is approximately canonically distributed has so far never been studied in detail.

The terms $-i / \hbar \hat{P}(t) \hat{L} \hat{\rho}_{P}(t)$ and $-i / \hbar \hat{P}(t) \hat{L} \int_{0}^{\infty} d s \ldots$ in the NZ-equation (3.39) can be physically interpreted as follows:

As we can see from Eq. (3.29), the term $-i / \hbar \hat{P}(t) \hat{L} \hat{\rho}_{P}(t)$ would yield the whole time-derivative $d \hat{\rho}_{P}(t) / d t$ if, at time $t$, the state of the system $\hat{\rho}(t)$ were entirely given by $\hat{\rho}_{P}(t)$. However, there is also the part described by $\hat{\rho}_{Q}(t)$, and thus there is the contribution $-i / \hbar \hat{P}(t) \hat{L} \hat{\rho}_{Q}(t)$ to the time-rate $d \hat{\rho}_{P}(t) / d t$. As has been shown, this term takes the form $-i / \hbar \hat{P}(t) \hat{L} \int_{0}^{\infty} d s \hat{G}(t, s) \hat{\sigma}(s)$. It describes that part of the rapid complicated time evolution which contributes to the change of the slowly time-dependent density operator $\hat{\rho}_{P}(t)$. As we shall see that this term describes friction and dissipation processes, we shall refer to it as the "dissipation term" of the NZ-equation.

As it stands, the NZ-equation (3.39) cannot be solved due to the complexity of the propagator $\hat{G}(t, s)$. As this propagator appears in the equation of motion of the slowly time-dependent density operator $\hat{\rho}_{P}(t)$, one may introduce some substantial simplifications, namely perturbation theory within the dissipative term and the Markov approximation.

Let us first define a "collective potential Hamiltonian" $\hat{H}_{\text {coll }}$ as a canonical average of the potential surfaces which correspond to the different intrinsic excitations of the system:

In Eq. (3.50), we first introduce a Hamiltonian $\hat{H}_{1}$ with the same eigenstates as the basis Hamiltonian $\hat{H}_{1}^{\prime}$ or $\hat{\mathcal{H}}^{\prime}$ resp., but with shifted eigenvalues

$$
\hat{H}_{1}:=\sum_{a q}|a q\rangle\left(\mathcal{E}_{a}^{\prime}(q)+\lambda(q)\langle a q|\hat{q}| a q\rangle\right)\langle a q|
$$


or, separately for the biorthogonal and the orthonormal basis, resp.,

$$
\begin{aligned}
& \hat{H}_{1}=\left\{\begin{array}{l}
\sum_{a q}\left|\phi_{a}(q)\right\rangle\left({E^{\prime}}_{a}^{(1)}(q)+\lambda(q)\left\langle\widetilde{\phi_{a}(q)}|\hat{q}| \phi_{a}(q)\right\rangle\right)\left\langle\widetilde{\phi_{a}(q)}\right| \\
\sum_{a q}\left|\Psi_{a}(q)\right\rangle\left(\varepsilon_{a}^{\prime}(q)+\lambda(q)\left\langle\Psi_{a}(q)|\hat{q}| \Psi_{a}(q)\right\rangle\right)\left\langle\Psi_{a}(q)\right|
\end{array}\right. \\
& \hat{H}_{1}|a q\rangle=\left(\mathcal{E}_{a}^{\prime}(q)+\lambda(q)\langle a q|\hat{q}| a q\rangle\right)|a q\rangle
\end{aligned}
$$

The reason for shifting the eigenvalues by the amount $\lambda(q)\langle a q|\hat{q}| a q\rangle$ is that $\hat{H}_{1}$ should be a simple part of the total Hamiltonian $\hat{H}$ rather than of the Routhian $\hat{H}^{\prime}=\hat{H}-\lambda \hat{q}$. We denote the new eigen-energies by

$$
\mathcal{E}_{a}(q):=\mathcal{E}_{a}^{\prime}(q)+\lambda(q)\langle a q|\hat{q}| a q\rangle
$$

The matrix-representation of $\hat{H}_{\text {coll }}$ is then defined as

$$
\left\langle a_{1} q_{1}\left|\hat{H}_{\text {coll }}\right| a_{2} q_{2}\right\rangle=\delta_{a_{1} a_{2}} \delta_{q_{1} q_{2}} \sum_{a} \mathcal{E}_{a}\left(q_{2}\right) F_{a}\left(q_{2}, T\left(q_{2}, t\right)\right)
$$

i.e. as the canonical mean value of the Hamiltonian $\hat{H}_{1}$. Due to the time-dependence of the temperature, the matrix $\left\langle a_{1} q_{1}\left|\hat{H}_{\text {coll }}\right| a_{2} q_{2}\right\rangle$ acquires a slow dependence on time.

The eigen-energies $\mathcal{E}_{a}(q)$ represent a family of potential surfaces, one for each quantum state $a$ of the intrinsic motion. As we have discussed in Section 2, the quantum number " $a$ " and the generator coordinate $q$ design a particular $\mathrm{HW}$-state $\phi_{a}(q)$, if the biorthogonal basis is used, and they denote the largest component $\phi_{a}(q)$ in the basis state $\Psi_{a}(q)$, if the orthonormal basis is used.

In the limit of vanishing temperature, the canonical mean value of the different potential surfaces becomes equal to the lowest potential surface $\mathcal{E}_{a_{0}}(q)$ :

$$
\lim _{T\left(q_{2}, t\right) \rightarrow 0} \sum_{a} \mathcal{E}_{a}(q) F_{a}(q, T)=\mathcal{E}_{a_{0}}(q)
$$

We define the coupling Hamiltonian $\hat{H}_{c p l}$ as the difference between the total Hamiltonian $\hat{H}$ and $\hat{H}_{c o l l}$ :

$$
\hat{H}_{c p l}=\hat{H}-\hat{H}_{\text {coll }}
$$

with the matrix-representation

$$
\left\langle a_{1} q_{1}\left|\hat{H}_{c p l}\right| a_{2} q_{2}\right\rangle=\left\langle a_{1} q_{1}|\hat{H}| a_{2} q_{2}\right\rangle-\left\langle a_{1} q_{1}\left|\hat{H}_{\text {coll }}\right| a_{2} q_{2}\right\rangle
$$

Using the Wick decomposition for $\hat{H}^{\prime}$, we obtain a more explicit form of $\left\langle a_{1} q_{1}\left|\hat{H}_{c p l}\right| a_{2} q_{2}\right\rangle$ :

$$
\left\langle a_{1} q_{1}\left|\hat{H}_{c p l}\right| a_{2} q_{2}\right\rangle=\left\langle a_{1} q_{1}\left|\hat{H}-\lambda\left(q_{2}\right) \hat{q}\right| a_{2} q_{2}\right\rangle+\lambda\left(q_{2}\right)\left\langle a_{1} q_{1}|\hat{q}| a_{2} q_{2}\right\rangle-\left\langle a_{1} q_{1}\left|\hat{H}_{\text {coll }}\right| a_{2} q_{2}\right\rangle
$$

or

$$
\left\langle a_{1} q_{1}\left|\hat{H}_{c p l}\right| a_{2} q_{2}\right\rangle=\delta_{a_{1} a_{2}} \delta_{q_{1} q_{2}}\left(\mathcal{E}_{a_{2}}^{\prime}\left(q_{2}\right)-\left\langle\left\langle\mathcal{E}^{\prime}\left(q_{2}\right)\right\rangle\right\rangle\right)+\lambda\left(q_{2}\right)\left(\left\langle a_{1} q_{1}|\hat{q}| a_{2} q_{2}\right\rangle-\left\langle\left\langle\hat{q}\left(q_{2}\right)\right\rangle\right\rangle\right)+\left\langle a_{1} q_{1}\left|\hat{H}_{2}^{(4)}\left(q_{2}\right)\right| a_{2} q_{2}\right\rangle
$$

Here, we have introduced a short notation for canonical mean values

$$
\begin{aligned}
& \left\langle\left\langle\mathcal{E}^{\prime}(q)\right\rangle\right\rangle=\sum_{a} \mathcal{E}_{a}^{\prime}(q) F_{a}(q, T(q, t)) \\
& \langle\langle\hat{q}(q)\rangle\rangle=\sum_{a}\langle a q|\hat{q}| a q\rangle F_{a}(q, T(q, t))
\end{aligned}
$$

The first term in Eq. (3.57) represents the fluctuation of the intrinsic energies around their thermal mean value at a given value of the g.c. $q_{2}$, and the second term the fluctuation of the "collective transport term" $\lambda\left(q_{2}\right)\left\langle a_{1} q_{1}|\hat{q}| a_{2} q_{2}\right\rangle$ 
around its thermal mean value at the g.c. $q_{2}$. The last term in Eq. (3.57) is given by the matrix-element of the 2-body part $\hat{H}_{2}^{(4)}\left(q_{2}\right)$ of the Hamiltonian $\hat{H}^{\prime}\left(q_{2}\right)$. The total matrix $\left\langle a_{1} q_{1}\left|\hat{H}_{c p l}\right| a_{2} q_{2}\right\rangle$ is hermitian if we use the orthonormal basis functions $\Psi_{a}(q)$ :

$$
\left\langle\Psi_{a_{1}}\left(q_{1}\right)\left|\hat{H}_{c p l}\right| \Psi_{a_{2}}\left(q_{2}\right)\right\rangle=\left\langle\Psi_{a_{2}}\left(q_{2}\right)\left|\hat{H}_{c p l}\right| \Psi_{a_{1}}\left(q_{1}\right)\right\rangle^{*}
$$

whereas the different terms on the r.h.s. of (3.57) are not hermitian. This is due to the fact that the Routhian $\hat{H}^{\prime}(q)=$ $\hat{H}-\lambda(q) \hat{q}$ depends on the value of the generator coordinate.

One can easily see that the Liouvillean $\hat{L}_{\text {coll }}$ associated with $\hat{H}_{\text {coll }}$ commutes with the projection operators: The matrix representation of the Liouvilleans read:

$$
\begin{aligned}
& L_{a_{1} q_{1} a_{2} q_{2} a_{3} q_{3} a_{4} q_{4}}=\delta_{a_{2} a_{4}} \delta_{q_{2} q_{4}}\left\langle a_{1} q_{1}|\hat{H}| a_{3} q_{3}\right\rangle-\delta_{a_{1} a_{3}} \delta_{q_{1} q_{3}}\left\langle a_{4} q_{4}|\hat{H}| a_{2} q_{2}\right\rangle
\end{aligned}
$$

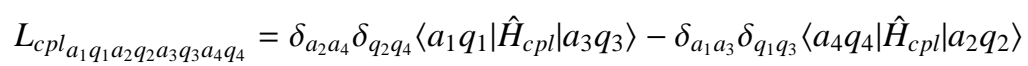

$$
\begin{aligned}
& L_{\text {coll } a_{1} q_{1} a_{2} q_{2} a_{3} q_{3} a_{4} q_{4}}:=\delta_{a_{2} a_{4}} \delta_{q_{2} q_{4}}\left\langle a_{1} q_{1}\left|\hat{H}_{\text {coll }}\right| a_{3} q_{3}\right\rangle-\delta_{a_{1} a_{3}} \delta_{q_{1} q_{3}}\left\langle a_{4} q_{4}\left|\hat{H}_{\text {coll }}\right| a_{2} q_{2}\right\rangle \\
& L_{\text {coll }_{1} q_{1} a_{2} q_{2} a_{3} q_{3} a_{4} q_{4}} \stackrel{(3.53)}{=} \delta_{a_{2} a_{4}} \delta_{q_{2} q_{4}} \delta_{a_{1} a_{3}} \delta_{q_{1} q_{3}}\left(\left\langle\left\langle\mathcal{E}\left(q_{1}\right)\right\rangle\right\rangle-\left\langle\left\langle\mathcal{E}\left(q_{2}\right)\right\rangle\right\rangle\right)
\end{aligned}
$$

Using the matrix-representations (3.16, 3.62), one easily checks that the following relations hold:

$$
\left[\hat{P}, \hat{L}_{\text {coll }}\right]=0=\left[1-\hat{Q}, \hat{L}_{\text {coll }}\right]=-\left[\hat{Q}, \hat{L}_{\text {coll }}\right]
$$

Due to the relations (3.63), the NZ-equation (3.39) can be written in the form:

$$
\begin{aligned}
\frac{d \hat{\rho}_{P}(t)}{d t}= & -\frac{i}{\hbar} \hat{P}(t)\left(\hat{L}_{c p l}(t) \hat{G}(t, 0) \hat{\rho}_{Q}(0)+\left(\hat{L}_{c o l l}(t)+\hat{L}_{c p l}(t)\right) \hat{\rho}_{P}(t)\right)-\frac{1}{\hbar^{2}} \hat{P}(t) \hat{L}_{c p l}(t) \int_{0}^{\infty} d s \hat{G}(t, s) \hat{Q}(s) \hat{L}_{c p l}(s) \hat{\rho}_{P}(s) \\
& +\frac{i}{\hbar} \hat{P}(t) \hat{L}_{c p l}(t) \int_{0}^{\infty} d s \hat{G}(t, s) \dot{\hat{P}}(s) \hat{\rho}_{P}(s)+\dot{\hat{P}}(t) \hat{\rho}_{P}(t)
\end{aligned}
$$

Eq. (3.64) is still rigorously valid. We shall now apply the "Markov approximation".

This approximation is based on the observation that the non-negligible contributions to the integrals in Eq. (3.64) originate from a short time interval $t-\tau_{r e l}<s \leq t$, where the "relaxation time" $\tau_{r e l}$ is much smaller than the typical time scale $\tau_{c}$ of the collective motion.

One may estimate $\tau_{r e l}$ to be between $10^{-23} \mathrm{~s}$ and $10^{-22} \mathrm{~s}$ and $\tau_{c}$ to be of the order of $10^{-21} \mathrm{~s}$, i.e.

$$
\tau_{r e l} \ll \tau_{c}
$$

The quantity $\hat{\rho}_{P}(t)$ and the temperature $T(q, t)$ (see Section 4) vary slowly, i.e. on the time scale $\tau_{c}$, and so do the projection operators $\hat{P}(t), \hat{Q}(t)$, and the Liouvillean $\hat{L}_{c p l}(t)$, which depend on time through the temperature. The Markov approximation consists of several steps:

1. The first step is to replace the time variable $s$ by $t$ in the slowly time-dependent quantities of the integrals:

$$
\begin{aligned}
& \int_{0}^{\infty} d s \hat{G}(t, s) \hat{Q}(s) \hat{L}_{c p l}(s) \hat{\rho}_{P}(s) \simeq \int_{0}^{\infty} d s \hat{G}(t, s) \hat{Q}(t) \hat{L}_{c p l}(t) \hat{\rho}_{P}(t) \\
& \int_{0}^{\infty} d s \hat{G}(t, s) \dot{\hat{P}}(s) \hat{\rho}_{P}(s) \simeq \int_{0}^{\infty} d s \hat{G}(t, s) \dot{\hat{P}}(t) \hat{\rho}_{P}(t)
\end{aligned}
$$

2. Simplification of the Green propagator $\hat{G}(t, s)$

Replacing the slowly time-dependent quantities $\hat{Q}(\tau)$ and $\dot{\hat{P}}(\tau)$ by $\hat{Q}(t)$ and $\dot{\hat{P}}(t)$ in $(3.40)$, the time-ordering $\hat{T}$ becomes superfluous and we obtain

$$
\hat{G}(t, s) \simeq \theta_{0}(t-s) \hat{Q}(t) e^{-\frac{i}{\hbar}(t-s)\left[\hat{L} \hat{Q}(t)-\frac{\hbar}{i} \dot{\hat{P}}(t)\right]}
$$


As the operator $\dot{\hat{P}}(t)$ is proportional to $\dot{T}$ and as the temperature varies slowly as a function of time, the change of the temperature during the relaxation time, i.e. $\tau_{r e l} \cdot \dot{T}(t)$ is a very small quantity. We, therefore, neglect the term proportional to $\dot{\hat{P}}(t)$ in $(3.68)$ altogether:

$$
\hat{G}(t, s) \simeq \theta_{0}(t-s) \hat{Q}(t) e^{-\frac{i}{\hbar}(t-s) \hat{L} \hat{Q}(t)}
$$

The form 3.69 ) of the propagator is still too complicated for a practical application because it contains the Liouvillean $\hat{L}$ of the total system.

There are two possibilities for further simplification: Either one considers the coupling Hamiltonian $\hat{H}_{c p l}$ as a perturbation, or one approximates the propagator (3.69) by considering the matrix elements of $\hat{H}_{c p l}$ as random numbers. The two ways of proceeding are in a certain sense complementary because the random matrix method implies that the interaction $\hat{H}_{c p l}$ acts many times.

In what follows, we shall use the perturbation theory for $\hat{H}_{c p l}$. In fact, perturbation theory underlies most of the work on transport processes and especially the work on nuclear fission [4, 19]. The random matrix method was used by Weidenmüller et al. [22] for formulating a transport theory of heavy ions reactions. It would be very interesting indeed to investigate the random matrix approximation of the propagator (3.69) parallely to the perturbation treatment. The formulation of the propagator (3.69) on the basis of the random matrix theory and the consequences thereof, for instance concerning the fluctuation-dissipation relation, would constitute a problem of its own merit, and exceed the scope of the present work.

The perturbation theory implies that we replace the total Liouvillean $\hat{L}$ in Eq. (3.69) by the Liouvillean $\hat{L}_{1}$ corresponding to the Hamiltonian $\hat{H}_{1}$ introduced in Eq. (3.50). One thus replaces the Green fuction (3.69) by

$$
\hat{G}(t, s) \simeq \theta_{0}(t-s) \hat{Q}(t) e^{-\frac{i}{\hbar}(t-s) \hat{L}_{1} \hat{Q}(t)}
$$

As the basis functions $|a q\rangle$ are eigenfunctions of the Hamiltonian $\hat{H}_{1}$ (see Eq. (3.51), the propagator (3.70) becomes very simple if we may replace the projection operator $\hat{Q}(t)$ in the exponent of (3.70) by 1 :

$$
\hat{G}(t, s) \simeq \theta_{0}(t-s) \hat{Q}(t) e^{-\frac{i}{\hbar}(t-s) \hat{L}_{1}}
$$

The replacement of Eq. (3.70) by Eq. (3.71) would be strictly valid if the projection operators $\hat{P}(t)$ and $\hat{Q}(t)$ commuted with $\hat{L}_{1}$. One easily checks the result

$$
\left(\left[\hat{P}, \hat{L}_{1}\right]\right)_{a_{1} q_{1} a_{2} q_{2} a_{3} q_{3} a_{4} q_{4}}=\delta_{a_{1} a_{2}} \delta_{a_{3} a_{4}} \delta_{q_{1} q_{3}} \delta_{q_{2} q_{4}} F_{a_{2}}\left(q_{2}\right)\left(\mathcal{E}_{a_{1}}\left(q_{2}\right)-\mathcal{E}_{a_{1}}\left(q_{1}\right)+\mathcal{E}_{a_{3}}\left(q_{1}\right)-\mathcal{E}_{a_{3}}\left(q_{2}\right)\right)
$$

It implies that the matrix element $\left\langle a_{1} q_{1}\left|\left[\hat{P}, \hat{L}_{1}\right]\right| a_{2} q_{2}\right\rangle$, and consequently also $\left\langle a_{1} q_{1}\left|\left[\hat{Q}, \hat{L}_{1}\right]\right| a_{2} q_{2}\right\rangle$ vanish for $q_{1}=q_{2}$. As the propagator $\hat{G}(t, s)$ appears in the "dissipative" integrals of Eq. (3.64), the contributions of the matrix-elements $\left\langle a_{1} q_{1}|\hat{G}(t, s)| a_{2} q_{2}\right\rangle$ with $q_{1}=q_{2}$ are expected to be much more important than the ones with $q_{1} \neq q_{2}$. As, within the subspace of matrix elements diagonal in the g.c., the Liouvillean $\hat{L}_{1}$ commutes with the projection operators, the approximation (3.71) of the expression (3.70) is acceptable.

Introducing the approximations (3.66), (3.67), and (3.71) into Eq. (3.64), the NZ-equation assumes the form :

$$
\begin{array}{r}
\frac{d \hat{\rho}_{P}(t)}{d t}=-\frac{i}{\hbar} \hat{P}(t)\left[\hat{L}_{c p l}(t) e^{-\frac{i}{\hbar} t \hat{L}_{1}} \hat{\rho}_{Q}(0)+\left(\hat{L}_{c o l l}(t)+\hat{L}_{c p l}(t)\right) \hat{\rho}_{P}(t)\right]+\dot{\hat{P}}(t) \hat{\rho}_{P}(t) \\
+\hat{P}(t) \hat{L}_{c p l}(t)\left[-\frac{1}{\hbar^{2}} \int_{0}^{\infty} d s \theta_{0}(t-s) \hat{Q}(t) e^{-\frac{i}{\hbar}(t-s) \hat{L}_{1}} \hat{L}_{c p l}(t) \hat{\rho}_{P}(t)\right. \\
\left.+\frac{i}{\hbar} \int_{0}^{\infty} d s \theta_{0}(t-s) \hat{Q}(t) e^{-\frac{i}{\hbar}(t-s) \hat{L}_{1}} \dot{\hat{P}}(t) \hat{\rho}_{P}(t)\right]
\end{array}
$$


Henceforth, we shall omit the inhomogeneous term in 3.73). Furthermore, in accordance with the approximation (3.71) of the Green function, we have put

$$
\hat{Q}(t) e^{-\frac{i}{\hbar}(t-s) \hat{L}_{1}} \hat{Q}(t) \simeq \hat{Q}(t) e^{-\frac{i}{\hbar}(t-s) \hat{L}_{1}}
$$

in the dissipative term of Eq. (3.73).

In the last term of Eq. (3.73) we may leave away the projection operator $\hat{Q}(t)$ making use of the identity (3.35). We can interpret this last term of (3.35) as a modification of the dissipative term due to the time-dependence of the temperature. As the temperature changes slowly as a function of time, we expect this term to be small.

We introduce the interaction representation of a Schrödinger operator $\hat{O}(t)$ by

$$
\hat{O}^{I}(s-t ; t)=e^{\frac{i}{\hbar}(s-t) \hat{L}_{1}} \hat{O}(t)=e^{\frac{i}{\hbar}(s-t) \hat{H}_{1}} \hat{O}(t) e^{-\frac{i}{\hbar}(s-t) \hat{H}_{1}}
$$

The explicit time-dependence on $t$ of the projection operators and of the Hamiltonian operators $\hat{H}_{c o l l}$ and $\hat{H}_{c p l}$ are produced by the variation in time of the temperature. Whereas the dependence of $\hat{O}^{I}(s-t ; t)$ on the second argument $t$ is slow, the dependence on $(s-t)$ is rapid, because it is related to the intrinsic excitations of the system. It is satisfactory that the two different time scales are seen to enter the equation of motion in a natural and automatic way:

$$
\begin{aligned}
\frac{d \hat{\rho}_{P}(t)}{d t} & =-\frac{i}{\hbar} \hat{P}(t)\left[\hat{H}_{c o l l}(t)+\hat{H}_{c p l}(t), \hat{\rho}_{P}(t)\right]+\dot{\hat{P}}(t) \hat{\rho}_{P}(t) \\
& -\frac{1}{\hbar^{2}} \hat{P}(t) \int_{0}^{\infty} d s \theta_{0}(t-s)\left[\hat{H}_{c p l}(t), \hat{Q}(t)\left[\hat{H}_{c p l}^{I}(s-t ; t), \hat{\rho}_{P}^{I}(s-t ; t)\right]\right] \\
& +\frac{i}{\hbar} \hat{P}(t) \int_{0}^{\infty} d s \theta_{0}(t-s)\left[\hat{H}_{c p l}(t), \hat{Q}(t) e^{i(s-t) \hat{H}_{1} / \hbar} \dot{\hat{P}}(t) \hat{\rho}_{P}(t) e^{-i(s-t) \hat{H}_{1} / \hbar}\right]
\end{aligned}
$$

Due to Eq. (3.34) and the relations $\hat{P}(t) \hat{Q}(t)=0$ and $\left[\hat{Q}(t), \hat{L}_{1}\right]=0$, we can simplify the two last terms of this equation. The NZ-equation for the density operator $\hat{\rho}_{P}(t)$ thus reads:

$$
\begin{aligned}
\frac{d \hat{\rho}_{P}(t)}{d t}= & -\frac{i}{\hbar} \hat{P}(t)\left[\hat{H}_{c o l l}(t)+\hat{H}_{c p l}(t), \hat{\rho}_{P}(t)\right]+\dot{\hat{P}}(t) \hat{\rho}_{P}(t) \\
& -\frac{1}{\hbar^{2}} \hat{P}(t) \int_{0}^{\infty} d s \theta_{0}(t-s) \hat{H}_{c p l}(t) \hat{Q}(t)\left[\hat{H}_{c p l}^{I}(s-t ; t), \hat{\rho}_{P}^{I}(s-t ; t)\right] \\
& +\frac{i}{\hbar} \hat{P}(t) \int_{0}^{\infty} d s \theta_{0}(t-s) \hat{H}_{c p l}(t) e^{i(s-t) \hat{H}_{1} / \hbar} \dot{\hat{P}}(t) \hat{\rho}_{P}(t) e^{-i(s-t) \hat{H}_{1} / \hbar}
\end{aligned}
$$

It is straightforward to derive from Eq. 3.76) an equation of motion for the reduced density matrix $R\left(q_{1}, q_{2} ; t\right)$ defined by Eq. (3.3): Writing (3.76) in terms of a matrix representation and using (3.42), we obtain

$$
\begin{aligned}
& \delta_{a_{1} a_{2}} F_{a_{2}}\left(q_{2}, T\left(q_{2}, t\right)\right) \sum_{a}\left\langle a q_{1}\left|\frac{d \hat{\rho}(t)}{d t}\right| a q_{2}\right\rangle=\delta_{a_{1} a_{2}} F_{a_{2}}\left(q_{2}, T\left(q_{2}, t\right)\right)\left\{-\frac{i}{\hbar} \sum_{a}\left\langle a q_{1}\left|\left[\hat{H}_{c o l l}(t)+\hat{H}_{c p l}(t), \hat{\rho}_{P}(t)\right]\right| a q_{2}\right\rangle\right. \\
& -\frac{1}{\hbar^{2}} \int_{0}^{\infty} d s \theta_{0}(t-s) \sum_{a}\left\langle a q_{1}\left|\hat{H}_{c p l}(t) \hat{Q}(t)\left[\hat{H}_{c p l}^{I}(s-t ; t), \hat{\rho}_{P}^{I}(s-t ; t)\right]\right| a q_{2}\right\rangle \\
& \left.+\frac{i}{\hbar} \int_{0}^{\infty} d s \theta_{0}(t-s) \sum_{a}\left\langle a q_{1}\left|\hat{H}_{c p l}(t) e^{i(s-t) \hat{H}_{1} / \hbar} \dot{\hat{P}}(t) \hat{\rho}_{P}(t) e^{-i(s-t) \hat{H}_{1} / \hbar}\right| a q_{2}\right\rangle\right\}
\end{aligned}
$$

Summing over $a_{1}, a_{2}$ and using the relation $\sum_{a} F_{a}(q, T(q, t))=1$, we find the following equation of motion for the 
reduced density matrix:

$$
\begin{aligned}
\frac{d R\left(q_{1}, q_{2} ; t\right)}{d t}= & -\frac{i}{\hbar} \sum_{a_{3} q_{3}}\left\{\mathcal{H}_{a_{3} a_{3}}\left(q_{1}, q_{3}\right) R\left(q_{3}, q_{2} ; t\right) F_{a_{3}}\left(q_{2}, T\left(q_{2}, t\right)\right)-R\left(q_{1}, q_{3} ; t\right) F_{a_{3}}\left(q_{3}, T\left(q_{3}, t\right)\right) \mathcal{H}_{a_{3} a_{3}}\left(q_{3}, q_{2}\right)\right\} \\
& -\frac{1}{\hbar^{2}} \int_{0}^{\infty} d s \theta_{0}(t-s) \sum_{a a_{3} q_{3} q_{4}} \mathcal{H}_{c p l a a_{3}}\left(q_{1} q_{3} ; t\right)\left\{\mathcal{H}_{c p l_{a_{3} a}}^{I}\left(q_{3} q_{4}\right) R_{a a}^{I}\left(q_{4} q_{2} ; s-t, t\right) F_{a}\left(q_{2}, T\left(q_{2}, t\right)\right)\right. \\
& -R_{a_{3} a_{3}}^{I}\left(q_{3} q_{4} ; s-t, t\right) F_{a_{3}}\left(q_{4}, T\left(q_{4}, t\right)\right) \mathcal{H}_{c p l}^{I}\left(l_{a_{3} a}\left(q_{4} q_{2}\right)\right\} \\
+ & \frac{1}{\hbar^{2}} \int_{0}^{\infty} d s \theta_{0}(t-s) \sum_{a a_{4} q_{3} q_{4}} \mathcal{H}_{c p l}\left(q_{1} q_{3} ; t\right) F_{a}\left(q_{2}, T\left(q_{2}, t\right)\right) \\
\times\left\{\mathcal{H}_{c p l_{a_{4} a_{4}}}^{I}\left(q_{3} q_{4}\right) R_{a_{4} a_{4}}^{I}\left(q_{4} q_{2} ; s-t, t\right) F_{a_{4}}\left(q_{2}, T\left(q_{2}, t\right)\right)-R_{a_{4} a_{4}}^{I}\left(q_{3} q_{4} ; s-t, t\right) F_{a_{4}}\left(q_{4}, T\left(q_{4}, t\right)\right) \mathcal{H}_{c p l_{a_{4} a_{4}}}^{I}\left(q_{4} q_{2}\right)\right\} & \\
& +\frac{i}{\hbar} \int_{0}^{\infty} d s \theta_{0}(t-s) \sum_{a_{3} q_{3}} \mathcal{H}_{c p l_{a_{3} a_{3}}}\left(q_{1} q_{3}\right) R_{a_{3} a_{3}}^{I}\left(q_{3} q_{2} ; s-t, t\right) \dot{F}_{a_{3}}\left(q_{2}, T\left(q_{2}, t\right)\right)
\end{aligned}
$$

Here, we have introduced the following notations:

$$
\begin{aligned}
& \mathcal{H}_{a_{1} a_{2}}\left(q_{1}, q_{2}\right):=\left\langle a_{1} q_{1}|\hat{H}| a_{2} q_{2}\right\rangle \\
& \mathcal{H}_{c p l_{a_{1} a_{2}}}\left(q_{1} q_{2} ; t\right):=\left\langle a_{1} q_{1}\left|\hat{H}_{c p l}(t)\right| a_{2} q_{2}\right\rangle \\
& \mathcal{H}_{c p l_{a_{1} a_{2}}}^{I}\left(q_{1} q_{2}\right):=\left\langle a_{1} q_{1}\left|\hat{H}_{c p l}^{I}(s-t ; t)\right| a_{2} q_{2}\right\rangle=e^{i(s-t) \omega_{a_{1} a_{2}}\left(q_{1}, q_{2}\right)}\left\langle a_{1} q_{1}\left|\hat{H}_{c p l}(t)\right| a_{2} q_{2}\right\rangle \\
& R_{a_{1} a_{2}}^{I}\left(q_{1} q_{2} ; s-t, t\right):=\left\langle a_{1} q_{1}\left|\hat{R}^{I}(s-t ; t)\right| a_{2} q_{2}\right\rangle=\delta_{a_{1} a_{2}} e^{i(s-t) \omega_{a_{1} a_{1}}\left(q_{1}, q_{2}\right)} R\left(q_{1}, q_{2} ; t\right) \\
& \hat{R}(t):=\sum_{a^{\prime} q_{1}^{\prime} q_{2}^{\prime}}\left|a^{\prime} q_{1}^{\prime}\right\rangle R\left(q_{1}^{\prime}, q_{2}^{\prime} ; t\right)\left\langle a^{\prime} q_{2}^{\prime}\right| \\
& \left\langle a_{1} q_{1}|\hat{R}(t)| a_{2} q_{2}\right\rangle=\delta_{a_{1} a_{2}} R\left(q_{1}, q_{2} ; t\right)
\end{aligned}
$$

The frequency $\omega_{a_{1} a_{2}}\left(q_{1}, q_{2}\right)$ occurring in Eq. (3.81) is related to the difference of eigen-energies (3.52) by

$$
\hbar \omega_{a_{1} a_{2}}\left(q_{1}, q_{2}\right):=\mathcal{E}_{a_{1}}\left(q_{1}\right)-\mathcal{E}_{a_{2}}\left(q_{2}\right)
$$

In the last term of Eq.(3.77), the factor $\dot{F}_{a_{3}}$ can be written in the form

$$
\dot{F}_{a_{3}}\left(q_{2}, T\left(q_{2}, t\right)\right)=\frac{\partial F_{a_{3}}\left(q_{2}, T\left(q_{2}, t\right)\right)}{\partial T} \dot{T}\left(q_{2}, t\right)
$$

The equation of motion 3.77) for the reduced density matrix $R\left(q_{1}, q_{2} ; t\right)$ must be solved together with the equation defining $T(q, t)$ which will be derived in the next Section.

\section{Determination of the temperature}

So far, the temperature entered the "ansatz" for $\hat{\rho}_{P}(t)$ as an undetermined parameter. If we had exact solutions for $\hat{\rho}_{P}(t)$ and $\hat{\rho}_{Q}(t)$, the choice of the temperature would not matter, as it would only concern the decomposition of $\hat{\rho}(t)$ into $\hat{\rho}_{P}(t)$ and $\hat{\rho}_{Q}(t)$. Of course, in reality, we determine $\hat{\rho}_{P}(t)$ from the equation (3.76), which was derived using the Markov approximation. One of the prerogatives of this approximation is that $\hat{\rho}_{P}(t)$ should depend slowly on time. Even more so, we require physically that $\hat{\rho}_{P}(t)$ should represent the slowly time-dependent part of the density operator $\hat{\rho}(t)$ "as accurately as possible". It is this requirement which must determine the choice of the temperature.

As the mean total energy of the system is independent of time, we should postulate in any case that the total energy of the system

$$
E:=\operatorname{Tr}(\hat{H} \hat{\rho}(t))=\operatorname{Tr}\left(\hat{H} \hat{\rho}_{P}^{h}(t)\right)
$$


is independent of time, where

$$
\hat{\rho}_{P}^{h}(t)=\frac{1}{2}\left(\hat{\rho}_{P}(t)+\hat{\rho}_{P}^{\dagger}(t)\right)
$$

is the hermitian part of $\hat{\rho}_{P}$.

The condition (4.1) was used in Ref. [4] to determine the temperature as a function of time. In addition, at any given time $t$, the temperature $T$ should also depend on the g.c. $q$, because the amount of intrinsic excitation is expected to vary along the dynamical paths of the system. In particular, we expect it to rise as the system approaches the scission region. Therefore, as a natural further condition to be fulfilled by the hermitian part $\hat{\rho}_{P}^{h}(t)$ of the reference density $\hat{\rho}_{P}(t)$, we require that it should yield the same average energy as the total density $\hat{\rho}(t)$ at any given value of the g.c. $q$.

In order that this condition involves only real functions, it is given in the form

$$
\frac{1}{2} \sum_{a} \operatorname{Re}\left\{\left\langle a q\left|[\hat{H}, \hat{\rho}(t)]_{+}\right| a q\right\rangle\right\}=\frac{1}{2} \sum_{a} \operatorname{Re}\left\{\left\langle a q\left|\left[\hat{H}, \hat{\rho}_{P}^{h}(t)\right]_{+}\right| a q\right\rangle\right\}
$$

The symmetrized forms $\frac{1}{2}[\hat{H}, \hat{\rho}]_{+}$and $\frac{1}{2}\left[\hat{H}, \hat{\rho}_{P}^{h}\right]_{+}$are used in (4.3) because the simple products $\hat{H} \hat{\rho}$ and $\hat{H} \hat{\rho}_{P}^{h}$ are not hermitian. In addition, we choose the real parts of the matrix elements because, in case of the biorthogonal basis, $\left\langle\widehat{\phi_{a}(q) \mid}[\hat{H}, \hat{\rho}(t)]_{+} \mid \phi_{a}(q)\right\rangle$ and $\left.\left\langle\widehat{\phi_{a}(q) \mid}\right| \hat{H}, \hat{\rho}_{P}^{h}(t)\right]_{+}\left|\phi_{a}(q)\right\rangle$ are not necessarily real.

Summing the Eq. (4.3) over the g.c. $q$ and using the completeness relation $\sum_{a q}|a q\rangle\langle a q|=1$, one finds that the Eq. (4.3) yields the condition (4.1) of a conserved mean value of the total energy.

The condition 4.3) can also be written in the form

$$
\sum_{a} \operatorname{Re}\left\{\left\langle a q\left|\left[\hat{H}, \hat{\rho}_{Q}^{h}(t)\right]_{+}\right| a q\right\rangle\right\}=0
$$

where $\hat{\rho}_{Q}^{h}(t)$ is defined by

$$
\hat{\rho}_{Q}^{h}(t):=\hat{\rho}(t)-\hat{\rho}_{P}^{h}(t)
$$

For the sake of simplicity, in Eq. (4.4), we replace the hermitian part $\hat{\rho}_{Q}^{h}(t)$ by $\hat{\rho}_{Q}(t)$ :

$$
\sum_{a} \operatorname{Re}\left\{\left\langle a q\left|\left[\hat{H}, \hat{\rho}_{Q}(t)\right]_{+}\right| a q\right\rangle\right\}=0
$$

If we use the orthonormal basis functions $\left(|a q\rangle=\left|\Psi_{a}(q)\right\rangle,\langle a q|=\left\langle\Psi_{a}(q)\right|\right)$, the conditions (4.4) and (4.6) are exactly equivalent, as one can show that

$$
\sum_{a} \operatorname{Re}\left\{\left\langle\Psi_{a}(q)\left|\left[\hat{H}, \hat{\rho}_{Q}^{a}(t)\right]_{+}\right| \Psi_{a}(q)\right\rangle\right\}=0
$$

where

$$
\hat{\rho}_{Q}^{a}(t)=\frac{1}{2}\left(\hat{\rho}_{Q}(t)-\hat{\rho}_{Q}^{\dagger}(t)\right)
$$

is the antihermitian part of $\hat{\rho}_{Q}(t)$.

If we use the biorthogonal basis functions $\left(|a q\rangle=\left|\phi_{a}(q)\right\rangle,\langle a q|=\left\langle\widetilde{\phi_{a}(q)}\right|\right)$, the condition (4.6) differs from (4.4), because

$$
\sum_{a} \operatorname{Re}\left\{\left\langle\widetilde{\phi_{a}(q)}\left|\left[\hat{H}, \hat{\rho}_{Q}^{a}(t)\right]_{+}\right| \phi_{a}(q)\right\rangle\right\} \neq 0
$$

If the spacing $\Delta q$ is such that the overlap parameter $\varepsilon \ll 1$, the expression (4.9), which is of $O(\varepsilon)$, is small. We thus expect that the simpler condition (4.6) yields almost the same temperature $T(q, t)$ as condition (4.4). Therefore, we, henceforth, use the relation (4.6) for determining the function $T(q, t)$.

Let us note that summing condition (4.6) over $q$ leads to the conservation of the total energy

$$
\operatorname{Re}\{\operatorname{Tr}(\hat{H} \hat{\rho}(t))\}=\operatorname{Re}\left\{\operatorname{Tr}\left(\hat{H} \hat{\rho}_{P}(t)\right)\right\}=\operatorname{Re}\left\{\operatorname{Tr}\left(\hat{H} \hat{\rho}_{P}^{h}(t)\right)\right\}
$$


as we have

$$
\operatorname{Re}\left\{\operatorname{Tr}\left(\hat{H} \hat{\rho}_{P}^{a}(t)\right)\right\}=\frac{1}{2}\left\{\operatorname{Tr}\left(\hat{H} \hat{\rho}_{P}^{a}(t)\right)+\operatorname{Tr}\left(\hat{H} \hat{\rho}_{P}^{a}(t)\right)^{\dagger}\right\}=0
$$

Let us now investigate the condition (4.6) in more detail:

The equation (3.37) presents the density operator $\hat{\rho}_{Q}(t)$ as a formal solution of the equation of motion (3.25). Applying the Markov approximation (3.71) to the Green operator $\hat{G}(t, s)$, leaving away the memory term $\hat{G}(t, 0) \hat{\rho}_{Q}(0)$, and replacing the source term $\hat{\sigma}(s)$ in Eq. (3.37) by $\hat{\sigma}(t)$, we obtain the density operator $\hat{\rho}_{Q}(t)$ in the form

$$
\hat{\rho}_{Q}(t) \simeq \int_{0}^{\infty} d s \theta_{0}(t-s) \hat{Q}(t) e^{-\frac{i}{\hbar}(t-s) \hat{L}_{1}} \hat{\sigma}(t)
$$

As for the condition 4.6

$$
\begin{aligned}
\operatorname{Re}\left\{\sum_{a}\left\langle a q\left|\left[\hat{H}, \hat{\rho}_{Q}(t)\right]_{+}\right| a q\right\rangle\right\}= & \operatorname{Re}\left\{\sum _ { a a _ { 1 } q _ { 1 } } \left(\left\langle a q|\hat{H}| a_{1} q_{1}\right\rangle\left\langle a_{1} q_{1}\left|\hat{\rho}_{Q}(t)\right| a q\right\rangle\right.\right. \\
& \left.\left.+\left\langle a q\left|\hat{\rho}_{Q}(t)\right| a_{1} q_{1}\right\rangle\left\langle a_{1} q_{1}|\hat{H}| a q\right\rangle\right)\right\}=0
\end{aligned}
$$

it is convenient to decompose $\hat{\rho}_{Q}(t)$ into two terms of different physical origin which correspond to the two terms of the source term (3.31):

$$
\begin{aligned}
& \hat{\rho}_{Q}(t)=\hat{\rho}_{Q}^{(1)}(t)+\hat{\rho}_{Q}^{(2)}(t) \\
& \hat{\rho}_{Q}^{(1)}(t):=-\frac{i}{\hbar} \int_{0}^{\infty} d s \theta_{0}(t-s) \hat{Q}(t) e^{-\frac{i}{\hbar}(t-s) \hat{L}_{1}} \hat{Q}(t) \hat{L}_{c p l}(t) \hat{\rho}_{P}(t) \\
& \hat{\rho}_{Q}^{(1)}(t) \simeq-\frac{i}{\hbar} \int_{0}^{\infty} d s \theta_{0}(t-s) \hat{Q}(t) e^{-\frac{i}{\hbar}(t-s) \hat{L}_{1}} \hat{L}_{c p l}(t) \hat{\rho}_{P}(t) \\
& \hat{\rho}_{Q}^{(2)}(t):=-\int_{0}^{\infty} d s \theta_{0}(t-s) e^{-\frac{i}{\hbar}(t-s) \hat{L}_{1}} \dot{\hat{P}}(t) \hat{\rho}_{P}(t)
\end{aligned}
$$

Replacing the form (4.15) of $\hat{\rho}_{Q}^{(1)}(t)$ by the form (4.16) implies the same approximation which was used to replace the form (3.70) of the Green operator by the simpler form (3.71). It is justified because, due to the factor $\left\langle a q|\hat{H}| a_{1} q_{1}\right\rangle$, the main contribution to the condition (4.13) arises for $q_{1}=q$. In the subspace of equal g.c., the projection operators commute with the Liouvillean $\hat{L}_{1}$, as one can see from Eq. (3.72).

After a straightforward calculation, one obtains

$$
\begin{aligned}
& \left\langle a_{1} q_{1}\left|\hat{\rho}_{Q}^{(1)}(t)\right| a q\right\rangle=-\frac{i}{\hbar} \int_{0}^{\infty} d s \theta_{0}(t-s)\left\{\left\langle a_{1} q_{1}\left|\left[\hat{H}_{c p l}^{I}(s-t, t), \hat{\rho}_{P}^{I}(s-t, t)\right]\right| a q\right\rangle\right. \\
& \left.-\delta_{a_{1} a} F_{a}(q, T(q, t)) \sum_{b}\left\langle b q_{1}\left|\left[\hat{H}_{c p l}^{I}(s-t, t), \hat{\rho}_{P}^{I}(s-t, t)\right]\right| b q\right\rangle\right\} \\
& \left\langle a_{1} q_{1}\left|\hat{\rho}_{Q}^{(2)}(t)\right| a q\right\rangle=-\int_{0}^{\infty} d s \theta_{0}(t-s) R_{a_{1} a}^{I}\left(q_{1}, q ; s-t, t\right) \frac{\partial F_{a}(q, T(q, t))}{\partial T} \dot{T}(q, t)
\end{aligned}
$$

where $\hat{H}_{c p l}^{I}(s-t, t)$ and $\hat{\rho}_{P}^{I}(s-t, t)$ are the operators $\hat{H}_{c p l}$ and $\hat{\rho}_{P}$ in the interaction picture (see Eq. (3.75), and where $R_{a_{1} a}^{I}\left(q_{1}, q ; s-t, t\right)$ is defined by Eq. (3.81).

The temperature occurs in the condition (4.13) with different generator coordinates as arguments. As the matrices of $\hat{\rho}_{Q}^{(1)}$ and $\hat{\rho}_{Q}^{(2)}$ are multiplied with matrix-elements $\mathcal{H}_{a a_{1}}\left(q, q_{1}\right)$ and $\mathcal{H}_{a_{1} a}\left(q_{1}, q\right)$ which exhibit a narrow Gaussian-like dependence on $q-q_{1}$, and as the "local" temperature $T\left(q_{1}, t\right)$ varies smoothly as a function of $q_{1}$, we may introduce the approximation of replacing all the temperature functions appearing in Eq. (4.13) by $T(q, t)$. 
Let us introduce the matrices $\mathcal{T}^{(1)}$ and $\mathcal{T}^{(2)}$ defined by putting all the $T$-functions occurring in the matrices $\hat{\rho}_{Q}^{(1)}$ and $\hat{\rho}_{Q}^{(2)}$ by $T(q, t)$

$$
\begin{aligned}
& \mathcal{T}_{a_{1} a}^{(1)}\left(q_{1}, q ; t\right):=\left\{\left\langle a_{1} q_{1}\left|\hat{\rho}_{Q}^{(1)}\right| a q\right\rangle\right\}_{\mathrm{a} l l T=T(q, t)} \\
& \mathcal{T}_{a_{1} a}^{(2)}\left(q_{1}, q ; t\right) \dot{T}(q, t):=\left\{\left\langle a_{1} q_{1}\left|\hat{\rho}_{Q}^{(2)}\right| a q\right\rangle\right\}_{\mathrm{a} l l T=T(q, t)}
\end{aligned}
$$

and correspondingly for the matrix-elements $\mathcal{T}_{a a_{1}}^{(1)}\left(q, q_{1} ; t\right)$ and $\mathcal{T}_{a a_{1}}^{(2)}\left(q, q_{1} ; t\right)$. As can be seen from Eq. (4.19), the r.h.s. of 4.21) reads more explicitely

$$
\mathcal{T}_{a_{1} a}^{(2)}\left(q_{1}, q ; t\right)=\left\{-\int_{0}^{\infty} d s \theta_{0}(t-s) R_{a_{1} a}^{I}\left(q_{1}, q ; s-t, t\right) \frac{\partial F_{a}(q, T)}{\partial T}\right\}_{T=T(q, t)}
$$

The condition (4.6) thus assumes the form

$$
\operatorname{Re} \sum_{a}\left(\left[\mathcal{H}, \mathcal{T}^{(1)}\right]_{+a a}(q, q)+\left[\mathcal{H}, \mathcal{T}^{(2)}\right]_{+a a}(q, q) \cdot \dot{T}(q, t)\right)=0
$$

or, more explicitely,

$$
\begin{aligned}
& \operatorname{Re}\left\{\sum_{a a_{1} q_{1}}\left(\mathcal{H}_{a a_{1}}\left(q, q_{1}\right) \mathcal{T}_{a_{1} a}^{(1)}\left(q_{1}, q ; t\right)+\mathcal{T}_{a a_{1}}^{(1)}\left(q, q_{1} ; t\right) \mathcal{H}_{a_{1} a}\left(q_{1}, q\right)\right)\right. \\
& \left.+\sum_{a a_{1} q_{1}}\left(\mathcal{H}_{a a_{1}}\left(q, q_{1}\right) \mathcal{T}_{a_{1} a}^{(2)}\left(q_{1}, q ; t\right)+\mathcal{T}_{a a_{1}}^{(2)}\left(q, q_{1} ; t\right) \mathcal{H}_{a_{1} a}\left(q_{1}, q\right)\right) \dot{T}(q, t)\right\}=0
\end{aligned}
$$

The temperature condition (4.24) and the equation of motion 3.77) represent a coupled system of equations for the reduced density matrix $R\left(q_{1}, q_{2} ; t\right)$ and the temperature $T(q, t)$. The solutions $R\left(q_{1}, q_{2} ; t\right)$ and $T(q, t)$ are uniquely determined whenever initial values $R\left(q_{1}, q_{2} ; t=0\right)$ and $T(q, t=0)$ are defined. As an example, one could assume that the initial reference density $\hat{\rho}_{P}(0)$ should correspond to a canonical distribution at the g.c. $q_{0}$ of the ground state valley. Then the initial values would be

$$
\begin{aligned}
& R\left(q_{1}, q_{2} ; 0\right)=\delta_{q_{1} q_{0}} \delta_{q_{2} q_{0}} \\
& \left\langle a_{1} q_{1}\left|\hat{\rho}_{P}(0)\right| a_{2} q_{2}\right\rangle=\delta_{q_{1} q_{0}} \delta_{q_{2} q_{0}} \delta_{a_{1} a_{2}} F_{a_{1}}\left(q_{0}, T\left(q_{0}, 0\right)\right)
\end{aligned}
$$

The initial temperature could be defined by the knowledge of the total energy $E_{t o t}$ :

$$
\sum_{a_{1}}\left\langle a_{1} q_{0}|\hat{H}| a_{1} q_{0}\right\rangle F_{a_{1}}\left(q_{0}, T\left(q_{0}, 0\right)\right)=E_{t o t}
$$

Let us comment the numerical problem of evaluating solutions of the coupled set of equations (3.77) for the reduced density $R\left(q_{1}, q_{2} ; t\right)$ and the equation (4.24) for the temperature $T(q, t)$ :

Given the fact that a realistic description of the fission process requires a set of 2 to 3 generator coordinates, the technical problem is one of storage capacity or of rapid subroutines. As already mentioned, we replace the continuous values of the g.c.s by a network of discrete values and we also discretize the time. The step lengths $\Delta q$ and $\Delta t$ are to be determined by the required accuracy.

From the knowledge of the initial functions $R\left(q_{1}, q_{2} ; t=0\right)$ and $T(q, t=0)$, we first obtain $R\left(q_{1}, q_{2} ; t=\Delta t\right)$ from Eq. (3.77) and then $T(q, \Delta t)$ from Eq. (4.24). Subsequently, at the $\mathrm{n}^{\text {th }}$ time step, one obtains $R\left(q_{1}, q_{2} ; t_{n}+\Delta t\right)$ from substituting $R\left(q_{1}, q_{2} ; t \leq t_{n}\right), T\left(q, t \leq t_{n}\right)$ on the r.h.s. of Eq. (3.77) and, analogously, one finds $T\left(q, t_{n}+\Delta t\right)$ from Eq. (4.24). If the storage capacity of the computer is sufficiently great, one can speed up the calculation considerably by storing the time-independent matrices $\left\langle a_{1} q_{1}\left|\hat{H}_{1}\right| a_{2} q_{2}\right\rangle,\left\langle a_{1} q_{1}\left|\hat{H}_{c p l}\right| a_{2} q_{2}\right\rangle$, and the eigenvalues $\varepsilon_{a}(q)$ before entering the routines for solving the Eqs. (3.77) and (4.24).

Through the Eqs. (3.77) and (4.24) the initial distribution $R\left(q_{1}, q_{2} ; t=0\right)$ is thus propagated through the potential 
landscape as a function of time up to the scission configurations which represent a $(\mathcal{N}-1)$ surface in the space of $\mathcal{N}$ generator coordinates. The propagation of the reduced density $R\left(q_{1}, q_{2} ; 0\right)$ up to values of $R\left(q_{1}, q_{2} ; t\right)$ on the "scission surface" is reminiscent of a percolation problem.

Let us conclude this Section with a few remarks concerning the entropy:

As a first step, we have to introduce an adequate definition of the entropy. It seems to be appropriate to define a "local" entropy $S(q, t)$ in relation to the "local" temperature $T(q, t)$ :

$$
\begin{aligned}
& S(q, T):=-\sum_{a} F_{a}(q, T) \ln F_{a}(q, T) \\
& S(q, T)=\frac{\left\langle\left\langle\mathcal{E}^{\prime}(q, T)\right\rangle\right\rangle-G(q, T)}{T}
\end{aligned}
$$

where

$$
\left\langle\left\langle\mathcal{E}^{\prime}(q, T)\right\rangle\right\rangle:=\sum_{a} \mathcal{E}_{a}^{\prime}(q) F_{a}(q, T)
$$

The total entropy $\mathrm{S}(\mathrm{t})$ of the system is then to be defined by summing $S(q, T)$ over all nuclear shapes weighted with the probability to find the system at the g.c. $q$ at time $t$ :

$$
S(t):=\sum_{q} S(q, T(q, t)) R(q, q ; t)
$$

We remind the reader of the fact (see Section 2 ) that $R(q, q ; t)$ can only be interpreted as a probability if we define it through the representation in terms of the orthonormal basis states $\Psi_{a}(q)$.

As long as we neglect the emission of light particles and photons during the fission process, we expect that the total entropy $S(t)$ rises as a function of time

$$
\frac{d S(t)}{d t}=\sum_{q} \frac{\partial S(q, T(q, t))}{\partial T} \dot{T}(q, t)+\sum_{q} S(q, T(q, t)) \dot{R}(q, q ; t) \geq 0
$$

As the process of creating an increasing amount of intrinsic excitations, which are simply described by a canonical distribution, corresponds to a loss of information, the entropy must rise. However, as the functions $T(q, t)$ and $R(q, q ; t)$ in (4.32) can only be obtained by solving the coupled equations (3.77) and (4.24), we cannot prove the statement (4.32) in generality.

In a realistic description of the fission process, deexcitation processes must be taken into account. As the emission processes reduce the entropy of the remaining system, the statement (4.32) can no longer be made.

\section{The fluctuation-dissipation relation}

The fluctuation-dissipation theorem relates part of the Fourier transforms of the response function and of the correlation function of a pair of operators with each other [3, 4, 5]. The response and correlation functions appear in a natural way when studying the response of a system in thermal equilibrium with regard to small perturbations. As we chose to treat the coupling Hamiltonian $\hat{H}_{c p l}$ in the integral term of the NZ-equation (3.77) as a perturbation, we may expect that some sort of fluctuation-dissipation relation should hold. As we do not deal with a thermal equilibrium, but with a slow passage of the system through thermal equilibrium states pertaining to different values of the g.c., we expect that a fluctuation-dissipation relation holds only in the limit of very slow collective transport.

Apart from the last term in Eq. (3.77), which depends on the time rate $\dot{T}$ of the temperature, the time integral in the Eq. (3.77) can be written as a sum of two terms

$$
\mathcal{D}\left(q_{1}, q_{2}, t\right):=\sum_{q_{1}^{\prime}} D_{1}\left(q_{1}, q_{2}, q_{1}^{\prime}, t\right) R\left(q_{1}^{\prime}, q_{2} ; t\right)+\sum_{q_{1}^{\prime} q_{2}^{\prime}} D_{2}\left(q_{1}, q_{2}, q_{1}^{\prime}, q_{2}^{\prime}, t\right) R\left(q_{1}^{\prime}, q_{2}^{\prime} ; t\right)
$$


where $D_{1}\left(q_{1}, q_{2}, q_{1}^{\prime}, t\right)$ and $D_{2}\left(q_{1}, q_{2}, q_{1}^{\prime}, q_{2}^{\prime}, t\right)$ are defined as follows

$$
\begin{aligned}
& D_{1}\left(q_{1}, q_{2}, q_{1}^{\prime}, t\right):=-\frac{1}{\hbar^{2}} \sum_{a a^{\prime} q^{\prime}} \int_{0}^{\infty} d s \theta_{0}(t-s) F_{a}\left(q_{2}, T\left(q_{2}, t\right)\right) e^{i(s-t) \omega_{a a}\left(q_{1}^{\prime}, q_{2}\right)} \\
& \times\left\{\mathcal{H}_{c p l} a_{a a^{\prime}}\left(q_{1}, q^{\prime} ; t\right) \mathcal{H}_{c p l_{a^{\prime} a}^{I}}^{I}\left(q^{\prime}, q_{1}^{\prime} ; s-t, t\right)-\mathcal{H}_{c p l} a_{a^{\prime} a^{\prime}}\left(q_{1}, q^{\prime} ; t\right) \mathcal{H}_{c p l}^{I}\left(q^{\prime}, q_{1}^{\prime} ; s-t, t\right) F_{a^{\prime}}\left(q_{2}, T\left(q_{2}, t\right)\right)\right\} \\
& D_{2}\left(q_{1}, q_{2}, q_{1}^{\prime}, q_{2}^{\prime}, t\right):=\frac{1}{\hbar^{2}} \sum_{a a^{\prime}} \int_{0}^{\infty} d s \theta_{0}(t-s) F_{a^{\prime}}\left(q_{2}^{\prime}, T\left(q_{2}^{\prime}, t\right)\right) e^{i(s-t) \omega_{a^{\prime} a^{\prime}}\left(q_{1}^{\prime}, q_{2}^{\prime}\right)} \\
& \times\left\{\mathcal{H}_{c p l a a^{\prime}}\left(q_{1}, q_{1}^{\prime} ; t\right) \mathcal{H}_{c p l_{a^{\prime} a}}^{I}\left(q_{2}^{\prime}, q_{2} ; s-t, t\right)-\mathcal{H}_{c p l a a}\left(q_{1}, q_{1}^{\prime} ; t\right) \mathcal{H}_{c p l_{a^{\prime} a^{\prime}}^{I}}\left(q_{2}^{\prime}, q_{2} ; s-t, t\right) F_{a}\left(q_{2}, T\left(q_{2}, t\right)\right)\right\}
\end{aligned}
$$

The matrix-elements of the coupling Hamimtonian $\hat{H}_{c p l}$ depend on the values of the generator coordinates in the bra and ket states through a narrow Gaussian (see Eqs. (1.16), (1.17). Thus, the matrix-elements have their largest values for equal values of the generator coordinates in bra and ket. Let us, therefore, consider the parts $D_{1}^{(0)}$ and $D_{2}^{(0)}$ of $D_{1}$ and $D_{2}$, which are of zeroth order in the overlap parameter $\varepsilon$ (see Eq. (2.6)):

$$
\begin{aligned}
& D_{1}^{(0)}\left(q_{1}, q_{2}, q_{1}^{\prime}, t\right)=-\frac{1}{\hbar^{2}} \sum_{a a^{\prime}} \int_{0}^{\infty} d s \theta_{0}(t-s) F_{a}\left(q_{2}, T\left(q_{2}, t\right)\right) e^{i(s-t) \omega_{a a}\left(q_{1}, q_{2}\right)}
\end{aligned}
$$

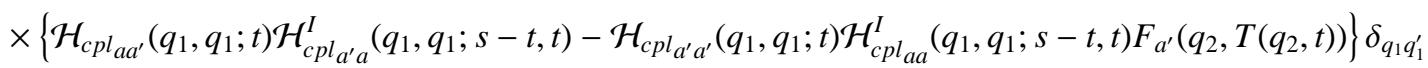

$$
\begin{aligned}
& D_{2}^{(0)}\left(q_{1}, q_{2}, q_{1}^{\prime}, q_{2}^{\prime}, t\right)=\frac{1}{\hbar^{2}} \sum_{a a^{\prime}} \int_{0}^{\infty} d s \theta_{0}(t-s) F_{a^{\prime}}\left(q_{2}, T\left(q_{2}, t\right)\right) e^{i(s-t) \omega_{a^{\prime} a^{\prime}}\left(q_{1}, q_{2}\right)} \\
& \times\left\{\mathcal{H}_{c p l_{a a^{\prime}}}\left(q_{1}, q_{1} ; t\right) \mathcal{H}_{c p l_{a^{\prime} a}^{I}}^{I}\left(q_{2}, q_{2} ; s-t, t\right)-\mathcal{H}_{c p l_{a a}}\left(q_{1}, q_{1} ; t\right) \mathcal{H}_{c p l_{a^{\prime} a^{\prime}}^{I}}^{I}\left(q_{2}, q_{2} ; s-t, t\right) F_{a}\left(q_{2}, T\left(q_{2}, t\right)\right)\right\} \delta_{q_{1}^{\prime} q_{1}} \delta_{q_{2}^{\prime} q_{2}}
\end{aligned}
$$

The exponential factors in (5.2) to (5.5) (for their definition, see Eqs. (3.81), (3.81') refer to differences of energies at different $q$-values $\left(q_{1}, q_{2}\right)$ on the same potentiel energy surface characterized by given quantum numbers of intrinsic excitation " $a$ " or " $a$ ".

In the special case $q_{1}=q_{2}$, the exponential factors are equal to 1 and the coefficients assume the simple form

$$
\begin{aligned}
D_{1}^{(0)}\left(q_{1}, q_{1}, q_{1}^{\prime}, t\right)=-\frac{1}{\hbar^{2}} \int_{0}^{\infty} d s \theta_{0}(t-s)\left\langle\left\langle\left(\mathcal{H}_{c p l}\left(q_{1}, q_{1}\right)-\left\langle\left\langle\mathcal{H}_{c p l}\left(q_{1}, q_{1}\right)\right\rangle\right\rangle_{q_{1}}\right)\right.\right. \\
\left.\left.\cdot\left(\mathcal{H}_{c p l}^{I}\left(q_{1}, q_{1}\right)-\left\langle\left\langle\mathcal{H}_{c p l}^{I}\left(q_{1}, q_{1}\right)\right\rangle\right\rangle_{q_{1}}\right)\right\rangle\right\rangle_{q_{1}} \delta_{q_{1} q_{1}^{\prime}} \\
D_{2}^{(0)}\left(q_{1}, q_{1}, q_{1}^{\prime}, q_{2}^{\prime}, t\right)=\frac{1}{\hbar^{2}} \int_{0}^{\infty} d s \theta_{0}(t-s)\left\langle\left\langle\left(\mathcal{H}_{c p l}^{I}\left(q_{1}, q_{1}\right)-\left\langle\left\langle\mathcal{H}_{c p l}^{I}\left(q_{1}, q_{1}\right)\right\rangle\right\rangle_{q_{1}}\right)\right.\right. \\
\left.\left.\cdot\left(\mathcal{H}_{c p l}\left(q_{1}, q_{1}\right)-\left\langle\left\langle\mathcal{H}_{c p l}\left(q_{1}, q_{1}\right)\right\rangle\right\rangle_{q_{1}}\right)\right\rangle\right\rangle_{q_{1}} \delta_{q_{1}^{\prime} q_{1}} \delta_{q_{2}^{\prime} q_{1}}
\end{aligned}
$$

where $\mathcal{H}_{c p l}$ and $\mathcal{H}_{c p l}^{I}$ signify the matrices

$$
\begin{aligned}
& \mathcal{H}_{c p l}\left(q_{1}, q_{2}\right):=\left\{\mathcal{H}_{c p l_{a_{1} a_{2}}}\left(q_{1}, q_{2} ; t\right)\right\} \\
& \mathcal{H}_{c p l}^{I}\left(q_{1}, q_{2}\right):=\left\{\mathcal{H}_{c p l_{a_{1} a_{2}}^{I}}\left(q_{1}, q_{2} ; s-t, t\right)\right\}
\end{aligned}
$$

and where we denote the thermal mean-values of an operator $\hat{O}$ at a given value of $q$ of the generator coordinates by

$$
\langle\langle\hat{O}\rangle\rangle_{q}:=\sum_{a} F_{a}(q, T(q, t))\langle a q|\hat{O}| a q\rangle
$$

The integrands on the right hand sides of the Eqs. (5.6), (5.7) are seen to represent the correlation between the fluctuations $\delta H_{c p l}(t), \delta H_{c p l}^{I}(s-t, t)$ of the operators $\hat{H}_{c p l}(t), \hat{H}_{c p l}^{I}(s-t, t)$ around their respective thermal mean values. Decomposing the product of the fluctuations into a commutator and an anticommutator, we may write the coefficients 
(5.6) and (5.7) in the form

$$
\begin{aligned}
& D_{1}^{(0)}\left(q_{1}, q_{1}, q_{1}^{\prime}, t\right)=-\frac{1}{2 \hbar^{2}} \int_{0}^{\infty} d s \theta_{0}(t-s)\left\{\left\langle\left\langle\left[\delta \mathcal{H}_{c p l}(t), \delta \mathcal{H}_{c p l}^{I}(s-t, t)\right]_{-}\right\rangle\right\rangle_{q}+\left\langle\left\langle\left[\delta \mathcal{H}_{c p l}(t), \delta \mathcal{H}_{c p l}^{I}(s-t, t)\right]_{+}\right\rangle\right\rangle_{q}\right\} \delta_{q_{1} q_{1}^{\prime}} \\
& D_{2}^{(0)}\left(q_{1}, q_{1}, q_{1}^{\prime}, q_{2}^{\prime}, t\right)=\frac{1}{2 \hbar^{2}} \int_{0}^{\infty} d s \theta_{0}(t-s)\left\{\left\langle\left\langle\left[\delta \mathcal{H}_{c p l}^{I}(s-t, t), \delta \mathcal{H}_{c p l}(t)\right]_{-}\right\rangle\right\rangle_{q}+\left\langle\left\langle\left[\delta \mathcal{H}_{c p l}^{I}(s-t, t), \delta \mathcal{H}_{c p l}(t)\right]_{+}\right\rangle\right\rangle_{q}\right\} \delta_{q_{1}^{\prime} q_{1}} \delta_{q_{2}^{\prime} q_{2}}
\end{aligned}
$$

The mean-values of the commutator parts of Eqs. (5.11) and (5.12) are proportional to the "response function" defined by

$$
\begin{aligned}
\chi\left(q, s^{\prime} ; t\right) & :=\frac{i}{\hbar} \sum_{a} \theta_{0}\left(-s^{\prime}\right) F_{a}(q, T(q, t))\left\langle a q\left|\left[\delta \hat{H}_{c p l}^{I}\left(s^{\prime}, t\right), \delta \hat{H}_{c p l}(t)\right]\right| a q\right\rangle \\
& =\frac{i}{\hbar} \theta_{0}\left(-s^{\prime}\right)\left\langle\left\langle\left[\delta \mathcal{H}_{c p l}^{I}\left(s^{\prime}, t\right), \delta \mathcal{H}_{c p l}(t)\right]_{-}\right\rangle\right\rangle_{q}
\end{aligned}
$$

Here, we used the following definitions

$$
\begin{aligned}
& s^{\prime}=s-t \\
& \delta \hat{H}_{c p l}^{I}\left(s^{\prime}, t\right):=\hat{H}_{c p l}^{I}\left(s^{\prime}, t\right)-\left\langle\left\langle\hat{H}_{c p l}^{I}\left(s^{\prime}, t\right)\right\rangle\right\rangle_{q} \\
& \delta \hat{H}_{c p l}(t):=\hat{H}_{c p l}(t)-\left\langle\left\langle\hat{H}_{c p l}(t)\right\rangle\right\rangle_{q}
\end{aligned}
$$

The slow time-dependence on $t$ is produced by the dependence of the temperature $T(q, t)$ on $t$. The time-dependence on $s^{\prime}$ is rapid due to the dependence on the intrinsic excitations of the system. The coefficients $D_{1}^{(0)}\left(q_{1}, q_{1}, q_{1}^{\prime}, t\right)$ and $D_{2}^{(0)}\left(q_{1}, q_{1}, q_{1}^{\prime}, q_{2}^{\prime}, t\right)$ being of very similar structure we, henceforth, shall refer to $D_{1}^{(0)}\left(q_{1}, q_{1}, q_{1}^{\prime}, t\right)$ only, as the results obtained for $D_{1}^{(0)}$ can be immediately transferred to $D_{2}^{(0)}$.

The purpose of the following investigation is to show that a fluctuation-dissipation relation holds for these parts of the coefficients $D_{1}$ and $D_{2}$.

To start with, we remind the reader of the fact that the coefficients $D_{1}^{(0)}$ and $D_{2}^{(0)}$ are defined by the parts of order $\varepsilon^{0}$ in the overlap parameter $\varepsilon$. Consequently, by consistency, we have to put

$$
\begin{aligned}
\left\langle a q\left|\delta \hat{H}_{c p l}^{I} \delta \hat{H}_{c p l}\right| a q\right\rangle & =\sum_{a^{\prime} q^{\prime}}\left\langle a q\left|\delta \hat{H}_{c p l}^{I}\right| a^{\prime} q^{\prime}\right\rangle\left\langle a^{\prime} q^{\prime}\left|\delta \hat{H}_{c p l}\right| a q\right\rangle \\
& \simeq \sum_{a^{\prime}}\left\langle a q\left|\delta \hat{H}_{c p l}^{I}\right| a^{\prime} q\right\rangle\left\langle a^{\prime} q\left|\delta \hat{H}_{c p l}\right| a q\right\rangle
\end{aligned}
$$

Let us introduce the Fourier transform $\widetilde{\chi}(q, \omega ; t)$ of the response function $\chi\left(q, s^{\prime} ; t\right)$

$$
\widetilde{\chi}(q, \omega ; t):=\int_{-\infty}^{+\infty} d s^{\prime} \chi\left(q, s^{\prime} ; t\right) e^{i \omega s^{\prime}},
$$

the so-called "dynamical susceptibility", and its "dissipative" part $\widetilde{\chi}^{d}(q, \omega ; t)$ defined by

$$
\left.\widetilde{\chi}^{d}(q, \omega ; t)=\frac{1}{2 i} \widetilde{\chi}(q, \omega ; t)-\widetilde{\chi}(q,-\omega ; t)\right)
$$

It is also given by

$$
\widetilde{\chi}^{d}(q, \omega ; t)=\int_{-\infty}^{+\infty} d s^{\prime} \chi\left(q, s^{\prime} ; t\right) \sin \left(\omega s^{\prime}\right)
$$

It is easily seen that $\widetilde{\chi}^{d}$ can be written in the form

$$
\tilde{\chi}^{d}(q, \omega ; t)=\frac{1}{2 \hbar} \int_{-\infty}^{+\infty} d s^{\prime} \sum_{a} F_{a}(q, T(q, t))\left\langle a q\left|\left[\delta \hat{H}_{c p l}^{I}\left(s^{\prime}, t\right), \delta \hat{H}_{c p l}(t)\right]_{-}\right| a q\right\rangle e^{i \omega s^{\prime}}
$$


The important point is that no Heaviside functions appear any more in the representation 5.21) of the dissipative susceptibility. Due to this feature, the r.h.s. of (5.21) is proportional to the Fourier transform of the antisymmetrical part $A\left(q, s^{\prime} ; t\right)$ of the function

$$
C\left(q, s^{\prime} ; t\right):=\sum_{a} F_{a}(q, T(q, t))\left\langle a q\left|\delta \hat{H}_{c p l}^{I}\left(s^{\prime}, t\right) \delta \hat{H}_{c p l}(t)\right| a q\right\rangle
$$

which represents the correlation between the fluctuations $\delta \hat{H}_{c p l}(t)=\delta \hat{H}_{c p l}^{I}(0, t)$ and $\delta \hat{H}_{c p l}^{I}\left(s^{\prime}, t\right)$.

Analogously to the decomposition of the expressions (5.11), (5.12), we write (5.22) in the form

$$
C\left(q, s^{\prime} ; t\right)=S\left(q, s^{\prime} ; t\right)+i A\left(q, s^{\prime} ; t\right)
$$

where the symmetrical and the antisymmetrical parts of the correlation function $C$ are defined by

$$
\begin{aligned}
& S\left(q, s^{\prime} ; t\right):=\frac{1}{2} \sum_{a} F_{a}(q, T(q, t))\left\langle a q\left|\left[\delta \hat{H}_{c p l}^{I}\left(s^{\prime}, t\right), \delta \hat{H}_{c p l}(t)\right]_{+}\right| a q\right\rangle \\
& A\left(q, s^{\prime} ; t\right):=\frac{1}{2 i} \sum_{a} F_{a}(q, T(q, t))\left\langle a q\left|\left[\delta \hat{H}_{c p l}^{I}\left(s^{\prime}, t\right), \delta \hat{H}_{c p l}(t)\right]_{-}\right| a q\right\rangle
\end{aligned}
$$

The Fourier transforms, sometimes referred to as "spectral functions", are given by

$$
\widetilde{C}(q, \omega ; t)=\int_{-\infty}^{+\infty} d s^{\prime} C\left(q, s^{\prime} ; t\right) e^{i \omega s^{\prime}}=\widetilde{S}(q, \omega ; t)+i \widetilde{A}(q, \omega ; t)
$$

where $\widetilde{S}(q, \omega ; t)$ and $\widetilde{A}(q, \omega ; t)$ are the Fourier transforms of $S(q, \omega ; t)$ and $A(q, \omega ; t)$. Comparing the r.h.s. of Eq. (5.21) with the definition of $\widetilde{A}(q, \omega ; t)$, one obtains the relation

$$
\widetilde{\chi}^{d}(q, \omega ; t)=\frac{i}{\hbar} \widetilde{A}(q, \omega ; t)
$$

which is one way of writing the fluctuation-dissipation relation.

In what follows, we shall show that the r.h.s. of Eq. (5.27) can be written in the form

$$
\frac{i}{\hbar} \widetilde{A}(q, \omega ; t)=\frac{1}{2 \hbar}\left(1-e^{-\beta \hbar \omega}\right) \widetilde{C}(q, \omega ; t)
$$

where $\beta(q, t)$ is the reciprocal temperature

$$
\beta(q, t):=\frac{1}{T(q, t)}
$$

The proof of the relation $(5.28$ is based on the "stationarity" of the mean-value

$$
\left\langle\left\langle\delta \hat{H}_{c p l}^{I}\left(s^{\prime}, t\right) \delta \hat{H}_{c p l}(t)\right\rangle\right\rangle_{q}=\left\langle\left\langle\delta \hat{H}_{c p l}(t) \delta \hat{H}_{c p l}^{I}\left(-s^{\prime}, t\right)\right\rangle\right\rangle_{q}
$$

and on the following symmetry relation for the correlation function

$$
C\left(q, s^{\prime} ; t\right)=C\left(q,-s^{\prime}-i \hbar \beta ; t\right)
$$

Although these two relations are well-known (see for instance Ref. [5]), we have to discuss their validity, which is not automatic in the context of our theory:

As a prerequisite of the proof, we have to accept the approximation 5.17) of only considering terms of order $\varepsilon^{0}$. We can thus use the cyclic invariance of the trace in a first step

$$
\begin{aligned}
\left\langle\left\langle\delta \hat{H}_{c p l}^{I}\left(s^{\prime}, t\right) \delta \hat{H}_{c p l}(t)\right\rangle\right\rangle_{q} & :=\sum_{a}\left\langle a q\left|\frac{e^{-\beta(q) \hat{H}_{1}^{\prime}(q)}}{Z_{0}(q)} e^{\frac{i}{\hbar} s^{\prime} \hat{H}_{1}} \delta \hat{H}_{c p l}(t) e^{-\frac{i}{\hbar} s^{\prime} \hat{H}_{1}} \delta \hat{H}_{c p l}(t)\right| a q\right\rangle \\
& =\sum_{a}\left\langle a q\left|e^{-\frac{i}{\hbar} s^{\prime} \hat{H}_{1}} \delta \hat{H}_{c p l}(t) \frac{e^{-\beta(q) \hat{H}_{1}^{\prime}(q)}}{Z_{0}(q)} e^{\frac{i}{\hbar} s^{\prime} \hat{H}_{1}} \delta \hat{H}_{c p l}(t)\right| a q\right\rangle
\end{aligned}
$$


ans, subsequently, the commutation property

$$
\left[\hat{H}_{1}, \hat{H}_{1}^{\prime}(q)\right]=0
$$

in a second step, for rewriting the r.h.s. of Eq. (5.32)

$$
=\sum_{a}\left\langle a q\left|\delta \hat{H}_{c p l}^{I}\left(-s^{\prime}, t\right) \frac{e^{-\beta(q) \hat{H}_{1}^{\prime}(q)}}{Z_{0}(q)} \delta \hat{H}_{c p l}(t)\right| a q\right\rangle=\left\langle\left\langle\delta \hat{H}_{c p l}(t) \delta \hat{H}_{c p l}^{I}\left(-s^{\prime}, t\right)\right\rangle\right\rangle_{q}
$$

This proves relation (5.30).

Let us now consider the explicit form of the function $C\left(q,-s^{\prime}-i \hbar \beta ; t\right)$

$$
C\left(q,-s^{\prime}-i \hbar \beta ; t\right)=\sum_{a}\left\langle a q\left|\frac{e^{-\beta(q) \hat{H}_{1}^{\prime}(q)}}{Z_{0}(q)} e^{\beta(q) \hat{H}_{1}} \delta \hat{H}_{c p l}^{I}\left(-s^{\prime}, t\right) e^{-\beta(q) \hat{H}_{1}} \delta \hat{H}_{c p l}(t)\right| a q\right\rangle
$$

If the Hamiltonian $\hat{H}_{1}^{\prime}(q)$, which appears in our equilibrium distribution, were equal to the Hamiltonian $\hat{H}_{1}$ defining the interaction picture, the r.h.s. of Eq. (5.34) would be equal to $C\left(q, s^{\prime}, t\right)$ because of the cyclic invariance.

In our case, these two Hamiltonians differ because we do not consider a true thermal equilibrium but a constrained one. In the expression (5.34), we may replace the operator $\hat{H}_{1}$ (see Eq. (3.50') by $\hat{H}_{1}(q)$, i.e. by the part of $\hat{H}_{1}$ acting on the subspace of states $|a q\rangle$ with fixed value $q$ :

$$
\hat{H}_{1}(q)=\sum_{a}|a q\rangle\left(\mathcal{E}_{a}^{\prime}(q)+\lambda(q)\langle a q|\hat{q}| a q\rangle\right)\langle a q| .
$$

The Hamiltonian $\hat{H}_{1}^{\prime}(q)$

$$
\hat{H}_{1}^{\prime}(q)=\sum_{a}|a q\rangle \mathcal{E}_{a}^{\prime}(q)\langle a q|
$$

differs from $\hat{H}_{1}(q)$ by $\lambda(q) \hat{q}(q)$

$$
\hat{H}_{1}(q)=\hat{H}_{1}^{\prime}(q)+\lambda(q) \hat{q}(q)
$$

$\hat{q}(q)$ being defined by

$$
\hat{q}(q):=\sum_{a^{\prime}}\left|a^{\prime} q\right\rangle\left\langle a^{\prime} q|\hat{q}| a^{\prime} q\right\rangle\left\langle a^{\prime} q\right|
$$

Eq. (5.34 can thus be written in the form

$$
\begin{aligned}
C\left(q,-s^{\prime}-i \hbar \beta ; t\right) & =\sum_{a}\left\langle a q\left|\frac{1}{Z_{0}(q)} e^{\beta(q) \lambda(q) \hat{q}(q)} \delta \hat{H}_{c p l}^{I}\left(-s^{\prime}, t\right) e^{-\beta(q)\left(\hat{H}_{1}^{\prime}(q)+\lambda(q) \hat{q}(q)\right)} \delta \hat{H}_{c p l}(t)\right| a q\right\rangle \\
& =\sum_{a}\left\langle a q\left|\delta \hat{H}_{c p l}^{I}\left(-s^{\prime}, t\right) \frac{e^{-\beta(q) \hat{H}_{1}^{\prime}(q)}}{Z_{0}(q)} e^{-\beta(q) \lambda(q) \hat{q}(q)} \delta \hat{H}_{c p l}(t) e^{\beta(q) \lambda(q) \hat{q}(q)}\right| a q\right\rangle
\end{aligned}
$$

In Eq. 5.39), the matrix-elements

$$
\left\langle a^{\prime} q\left|e^{-\beta(q) \lambda(q) \hat{q}(q)} \delta \hat{H}_{c p l}(t) e^{\beta(q) \lambda(q) \hat{q}(q)}\right| a q\right\rangle=\left\langle a^{\prime} q\left|\hat{H}_{c p l}(t)\right| a q\right\rangle e^{-\beta(q) \lambda(q)\left(\left\langle a^{\prime} q|\hat{q}| a^{\prime} q\right\rangle-\langle a q|\hat{q}| a q\rangle\right)}
$$

appear. The symmetry (5.31) of the correlation function $C$ then holds, if we may put the exponential factor approximately equal to 1

$$
e^{-\beta(q) \lambda(q)\left(\left\langle a^{\prime} q|\hat{q}| a^{\prime} q\right\rangle-\langle a q|\hat{q}| a q\rangle\right)} \simeq 1
$$

The approximation (5.40) holds, if the multipole moments in different intrinsic excitations do not fluctuate too much.

Let us assume that the approximation (5.40) is valid. We then claim that the following relation holds for the Fourier transforms of the correlation function

$$
\widetilde{C}(q, \omega ; t)=\widetilde{C}(q,-\omega ; t) e^{\beta(q) \hbar \omega}
$$


as a result of the symmetry property 5.31 . The proof is straightforward:

$$
\begin{aligned}
\widetilde{C}(q, \omega ; t) & =\int_{-\infty}^{+\infty} d s^{\prime} C\left(q, s^{\prime} ; t\right) e^{i \omega s^{\prime}} \stackrel{5.31}{=} \int_{-\infty}^{+\infty} d s^{\prime} C\left(q,-s^{\prime}-i \hbar \beta ; t\right) e^{i \omega s^{\prime}} \\
& =\int_{-\infty-i \hbar \beta}^{+\infty-i \hbar \beta} d s^{\prime \prime} C\left(q, s^{\prime \prime} ; t\right) e^{-i \omega s^{\prime \prime}} e^{\beta(q) \hbar \omega}=\widetilde{C}(q,-\omega ; t) e^{\beta(q) \hbar \omega}
\end{aligned}
$$

As an immediate consequence of the relation (5.41) we obtain

$$
\begin{aligned}
& \widetilde{S}(q, \omega ; t)=\frac{1}{2}\left(1+e^{-\beta(q) \hbar \omega}\right) \widetilde{C}(q,-\omega ; t) \\
& \widetilde{A}(q, \omega ; t)=\frac{1}{2 i}\left(1-e^{-\beta(q) \hbar \omega}\right) \widetilde{C}(q,-\omega ; t)
\end{aligned}
$$

Substituting the result (5.43) into Eq. (5.27), we find the conventional form

$$
\widetilde{\chi}^{d}(q, \omega ; t)=\frac{1}{2 \hbar}\left(1-e^{-\beta(q) \hbar \omega}\right) \widetilde{C}(q,-\omega ; t)
$$

of the fluctuation-dissipation relation [5].

In Section 1, we had mentioned the simple classical result of Einstein (see Eq. 1.1p) relating the diffusion coefficient $D$ to the friction constant $\gamma$ for the case of Brownian particles of mass $M$ immersed into a gaseous or liquid medium.

The classical limit of the relation (5.44) is obtained in the limit

$$
\beta(q, t) \hbar \omega=\frac{\hbar \omega}{T(q, t)} \ll 1
$$

In the approximation

$$
e^{-\beta \hbar \omega} \simeq 1-\beta \hbar \omega
$$

the fluctuation-dissipation relation (5.44) assumes the form

$$
\widetilde{C}(q,-\omega ; t) \simeq \frac{2 T}{\omega} \widetilde{\chi}^{d}(q, \omega ; t)
$$

It does no longer contain explicitly Planck's constant and corresponds to the Einstein relation (1.1).

Using Eq. (5.42), one can also relate the Fourier transform $\bar{S}(q, \omega ; t)$ to $\widetilde{\chi}^{d}(q, \omega ; t)$ :

$$
\widetilde{S}(q, \omega ; t)=\hbar \operatorname{coth}\left(\frac{\beta(q, t) \hbar \omega}{2}\right) \widetilde{\chi}^{d}(q, \omega ; t)
$$

with the classical limit

$$
\widetilde{S}(q, \omega ; t) \simeq \frac{2 T}{\omega} \widetilde{\chi}^{d}(q, \omega ; t)
$$

It is thus shown that the parts of order $\mathrm{O}\left(\varepsilon^{0}\right)$ of the coefficients $D_{1}$ and $D_{2}$ (eqs. (5.2), (5.3) ) do fulfill a fluctuationdissipation relation. This is expected from the fact that in the limit $\mathrm{O}\left(\varepsilon^{0}\right)$, the coefficients $D_{1}$ and $D_{2}$ do not describe a transport of the system between different values of the generator coordinates but rather a quasi-stationary equilibrium at a given value of the generator coordinates.

\section{Simple limits of the theory}

Let us consider the equation of motion (3.77) for the reduced density matrix $R\left(q_{1}, q_{2} ; t\right)$ in some special cases: 
If we restrict the Hill-Wheeler space to the grounstates $\phi_{a_{0}}(q)$ at each given value of the g.c. $q$, eq. (3.77) assumes the simple form

$$
\frac{d R\left(q_{1}, q_{2} ; t\right)}{d t}=-\frac{i}{\hbar} \sum_{q_{3}}\left[\mathcal{H}_{a_{0} a_{0}}\left(q_{1}, q_{3}\right) R\left(q_{3}, q_{2} ; t\right)-R\left(q_{1}, q_{3} ; t\right) \mathcal{H}_{a_{0} a_{0}}\left(q_{3}, q_{2}\right)\right]
$$

This equation is equivalent to the description of the fission process by the usual GCM without intrinsic excitations. The latter theory underlies the calculations of Refs. [9, 10]. Using this theory, it was found that, at small excitation energies $(\lesssim 10 \mathrm{MeV})$, the calculated mass distribution of the fragments in ${ }^{238} \mathrm{U}$ agreed quite well with the experimental one, although its width was a little too small [10]. In this respect, it should be noted that the omission of intrinsic excitations does not mean that vibrations of the nucleus shape on the way to scission are neglected. On the contrary, the dynamical role of these vibrations was proved to be important to explain the large width of the calculated mass distribution.

Another approximate version of the equation of motion (3.77) is obtained in the case that the "local" temperature $T(q, t)$ is small compared to the energy of the lowest elementary excitation $a \neq a_{0}$ :

$$
T(q, t) \ll \operatorname{Min}\left(\mathcal{E}_{a}^{\prime}(q)-\mathcal{E}_{a_{0}}^{\prime}(q)\right)
$$

The Boltzmann factors $F_{a}(q, T)$ can be written in the form

$$
F_{a}(q, T)=\frac{e^{-\mathcal{E}_{a}^{\prime}(q) / T}}{Z_{0}(q, T)}=\frac{e^{-\left(\mathcal{E}_{a}^{\prime}(q)-\mathcal{E}_{a_{0}}^{\prime}(q)\right) / T}}{1+\sum_{b \neq a_{0}} e^{-\left(\mathcal{E}_{b}^{\prime}(q)-\mathcal{E}_{a_{0}}^{\prime}(q)\right) / T}}
$$

which, in the limit of Eq. (6.2), yields

$$
F_{a}(q, T) \simeq \delta_{a a_{0}}
$$

Using the same technique, it is easy to verify that the time-derivative $\dot{F}_{a}=\dot{T} \partial F / \partial T$ goes to zero in the same limit. If we substitute these limiting values into 3.77), we obtain

$$
\begin{array}{r}
\frac{d R\left(q_{1}, q_{2} ; t\right)}{d t}=-\frac{i}{\hbar} \sum_{q_{3}}\left[\mathcal{H}_{a_{0} a_{0}}\left(q_{1}, q_{3}\right) R\left(q_{3}, q_{2} ; t\right)-R\left(q_{1}, q_{3} ; t\right) \mathcal{H}_{a_{0} a_{0}}\left(q_{3}, q_{2}\right)\right] \\
-\frac{1}{\hbar^{2}} \int_{0}^{\infty} d s \theta_{0}(t-s) \sum_{q_{3} q_{4}} \sum_{a \neq a_{0}}\left\{\mathcal{H}_{c p l_{a_{0} a}}\left(q_{1} q_{3} ; t\right) \mathcal{H}_{c p l_{a a_{0}}}^{I}\left(q_{3} q_{4} ; s-t, t\right) e^{i \omega_{a_{0} a_{0}}\left(q_{4}, q_{2}\right)(s-t)} R\left(q_{4}, q_{2} ; t\right)\right. \\
\left.-\mathcal{H}_{c p l_{a a_{0}}}\left(q_{1} q_{3} ; t\right) \mathcal{H}_{c p l_{a_{0} a}}^{I}\left(q_{4} q_{2} ; s-t, t\right) e^{i \omega_{a_{0} a_{0}}\left(q_{3}, q_{4}\right)(s-t)} R\left(q_{3}, q_{4} ; t\right)\right\}
\end{array}
$$

It is interesting to observe that, in the limit (6.2), our equation of motion (6.5) still contains terms describing "virtual" transitions to intrinsically excited states $|a q\rangle \neq\left|a_{0} q\right\rangle$. Since the two terms involving these transitions are of similar structure but of different sign, we do not expect a large contribution from them. This is no longer expected whenever the temperature $T(q, t)$ is no longer small compared to the elementary excitations. In this case, the equation of motion (3.77) must be solved as it stands, together with the eq. (4.24) which determines the temperature $T(q, t)$.

One notices from Eq. (6.3) that the Boltzmann factor $F_{a}(q, t)$ becomes very small whenever the intrinsic excitation energy $\mathcal{E}_{a}^{\prime}(q)-\mathcal{E}_{a_{0}}^{\prime}(q)$ is much larger than the temperature $T(q, t)$. This means that, in the equation of motion (3.77), only those intrinsic excitations $|a q\rangle$ need to be taken into account which correspond to non-negligible Boltzmann factors $F_{a}(q, t)$.

The actual computing work concerns the calculation of the matrix-elements $\mathcal{H}_{a a}\left(q, q^{\prime}\right)$ and $\mathcal{H}_{c p l a a^{\prime}}\left(q, q^{\prime}\right)$, on the one hand, and the numerical solution of the integro-differential equation (3.77), on the other. One could convert the integral part of the equation into partial differential terms of the Fokker-Planck type by a moment expansion of the integral kernels. We do not think that this is useful, because the moment expansion introduces approximations and the resulting partial differential equation for $R\left(q_{1}, q_{2} ; t\right)$ is also difficult to solve numerically. Thus we think that a straightforward numerical solution of Eq. (3.77) on the basis of a discretization of the g.c. $q$ is the best way to carry out practical applications of the theory. 
Let us add that the techniques used in earlier calculations where intrinsic excitations were omitted [9, 10] could be employed also in actual applications of the present theory. For instance, in fission problems, it will probably be convenient to define a "scission region" beyond which fission fragments no longer exchange nucleons or internal energy and are subject only to Coulomb repulsion and decay. Then fragments characteristics such as mass-, chargeor kinetic energy distributions (prior to neutron and $\gamma$-ray emission) could be evaluated from the values taken at large times by the solution of the equation of motion (3.77) obtained in the scission region or slightly beyond.

\section{Summary and Discussion}

a) Short review of what has been done

The basis of our work is the method proposed by Hill and Wheeler [8] of expanding the nuclear many-body state in terms of a set of basis functions which depend parametrically on deformation variables $q$ (see Eq. (1.4) which are integrated over. In this way, the description of the nuclear shape and of its dynamical change as a function of time does not necessitate the introduction of superfluous degrees of freedom.

This method which has been successfully applied to the description of single nuclear states has been applied to the nuclear density operator and its time evolution in the present paper.

We used as basis functions states of independently moving quasi-particles defined as eigenstates $\phi_{a}(q)$ of the one-body part of $\hat{H}_{1}^{\prime}(q)$ of the constrained Hartree-Fock-Bogoliubov (HFB) Hamiltonian (see Eq. (1.10)). It is a most welcome property of these states that, in the limit of vanishing collective large scale motion, they represent a self-consistently determined state of equilibrium at each given shape of the nucleus.

A complication is produced by the non-orthogonality of these states. We coped with it by either using a biorthogonal set of basis functions (see Eq. (1.15) and Section 2) or by introducing an orthogonalized version of the HillWheeler states (see Eqs. (2.11), (2.12)).

The basic assumptions of our theory are the ansatz 3.7 for the slowly time-dependent part $\hat{\rho}_{P}(t)$ of the statistical operator and the Markov approximation (see text after Eq. (3.64) simplifying the equation of motion (3.64) for $\hat{\rho}_{P}(t)$. The physical idea underlying the choice of $\hat{\rho}_{P}(t)$ is that this slowly time-dependent part of the statistical operator depends neither on the detailed phase relations of the rapidly time-depending part of $\hat{\rho}(t)$ nor on the exact occupation amplitudes of the intrinsic excitations at a given shape of the nucleus which thus may be represented by a canonical distribution (see Eq. (3.8)).

In Section 4, the temperature $T$ which occurs in the canonical distribution is chosen by the requirement that the reference density $\hat{\rho}_{P}(t)$ should correctly describe the total mean energy (see Eq. (4.1) ) and the energy at a given shape $q$ of the nucleus (see Eq. 4.3) at all times.

An important question is whether our theory implies a relation between the quantities describing the dissipation and fluctuation in the equation of motion (3.76) for $\hat{\rho}_{P}(t)$. We dealt with this question in Section 5 .

b) Short discussion of what should still be done

Obviously, a pertinent question is how one is to describe the emission of nucleons and photons during the fission process. We note that the emission during the dynamical process of fission is more difficult to treat than the emission from the fragments which have reached a thermal equilibrium. We are working on a description of emission processes during fission in the framework of our theory. The work will be submited for publication at our earliest convenience.

A very important endeavour will be to perform actual numerical calculations on the basis of the presented theory. Indeed, it is one of the purposes of the microscopic theory to find out whether we are going to find a satisfactory agreement between calculated and measured results on the fission process. This is by no means obvious because not only the assumptions of our theory are at stake but also the very concept of whether it is at all possible to describe all the low energy phenomena of nuclear physics on the basis of one given effective nucleon-nucleon interaction. This implication is a much farther going assumption than the hypothesis of the existence of an energy-density functional enabling us to calculate ground state properties.

It should be noted that calculations on the fission process at very low excitation energy have already been performed within the generator coordinate method without inclusion of intrinsic excitations [10] where an encouragingly good agreement between calculated and measured results has been found. The present theory would give the possibility to extend these calculations to higher excitation energy and compare them with the existing careful experimental work. 
Finally, a problem of its own merit would be to investigate in detail the classical limit of the theory presented. It surely is related to the $\delta$-function limit of the narrow gaussian overlap of many-body wavefunctions pertaining to different values of the generator coordinates. This fact suggests that the Fokker-Planck approximation of our equation of motion (3.77) for the reduced density $R\left(q_{1}, q_{2} ; t\right)$ might be the appropriate starting point for this investigation.

\section{Acknowledgements}

The collaboration between the authors of this paper was made possible by the enduring hospitality extended to one of us (K.D.) by the Centre DAM Île de France de Bruyères-le-Châtel. K.D. expresses his gratitude to the Centre.

\section{Appendices}

\section{A. Some properties of the projection operators}

In Eq. (3.16), we have introduced the matrix-representation of the projection $\hat{P}$. As the temperature $T$ depends on time, the operator $\dot{\hat{P}}$ is unequal from 0 and its matrix-representation is

$$
\dot{\hat{P}}_{a_{1} q_{1} a_{2} q_{2} a_{3} q_{3} a_{4} q_{4}}=\delta_{a_{1} a_{2}} \delta_{q_{1} q_{3}} \delta_{q_{2} q_{4}} \delta_{a_{3} a_{4}} \dot{F}_{a_{2}}\left(q_{2}, T\left(q_{2}, t\right)\right)
$$

The matrix-representation of $\hat{P} \dot{\hat{P}}$ is thus obtained as

$$
\begin{aligned}
& \sum_{a_{3} q_{3} a_{4} q_{4}} \hat{P}_{a_{1} q_{1} a_{2} q_{2} a_{3} q_{3} a_{4} q_{4}} \dot{\hat{P}}_{a_{3} q_{3} a_{4} q_{4} a_{5} q_{5} a_{6} q_{6}}= \\
& \delta_{a_{1} a_{2}} \delta_{q_{1} q_{5}} \delta_{q_{2} q_{6}} \delta_{a_{5} a_{6}} F_{a_{2}}\left(q_{2}, T\left(q_{2}, t\right)\right) \sum_{b} \dot{F}_{b}\left(q_{2}, T\left(q_{2}, t\right)\right)=0
\end{aligned}
$$

where the r.h.s. vanishes because we have

$$
\sum_{b} F_{b}\left(q_{2}, T\left(q_{2}, t\right)\right)=1
$$

On the other hand, for the matrix-representation of $\dot{\hat{P}} \hat{P}$, one finds

$$
\begin{aligned}
\sum_{a_{3} q_{3} a_{4} q_{4}} & \dot{\hat{P}}_{a_{1} q_{1} a_{2} q_{2} a_{3} q_{3} a_{4} q_{4}} \hat{P}_{a_{3} q_{3} a_{4} q_{4} a_{5} q_{5} a_{6} q_{6}}= \\
& =\delta_{a_{1} a_{2}} \delta_{q_{1} q_{5}} \delta_{q_{2} q_{6}} \delta_{a_{5} a_{6}} \dot{F}_{a_{2}}\left(q_{2}, T\left(q_{2}, t\right)\right) \sum_{b} F_{b}\left(q_{2}, T\left(q_{2}, t\right)\right) \\
& =\dot{\hat{P}}_{a_{1} q_{1} a_{2} q_{2} a_{5} q_{5} a_{6} q_{6}}
\end{aligned}
$$

The results (A.2), and (A.3) can be written in the form

$$
\begin{aligned}
& \hat{P}(t) \dot{\hat{P}}(t)=0 \\
& \dot{\hat{P}}(t) \hat{P}(t)=\dot{\hat{P}}(t)
\end{aligned}
$$

respectively. As we have

$$
\dot{\hat{Q}}=-\dot{\hat{P}}
$$

as a consequence of Eq. (3.17), we find

$$
\begin{aligned}
& \hat{P}(t) \dot{\hat{Q}}(t)=0=(1-\hat{Q}(t)) \dot{\hat{Q}}(t) \\
& \dot{\hat{Q}}(t) \hat{P}(t)=\hat{Q}(t)=\dot{\hat{Q}}(t)(1-\hat{Q}(t))
\end{aligned}
$$


from (A.4), and (A.5). Eqs. A.7 and A.8 imply the relations

$$
\begin{aligned}
& \hat{Q}(t) \dot{\hat{Q}}(t)=\dot{\hat{Q}}(t) \\
& \hat{\hat{Q}}(t) \hat{Q}(t)=0 \\
& \hat{Q}(t) \dot{\hat{P}}(t)=\dot{\hat{P}}(t) \\
& \hat{\hat{P}}(t) \hat{Q}(t)=0
\end{aligned}
$$

For any given operator $\hat{A}$, the matrix-representation of $\dot{\hat{P}} \hat{A}$ reads

$$
\begin{aligned}
\left\langle a_{1} q_{1}|\dot{\hat{P}}(t) \hat{A}| a_{2} q_{2}\right\rangle & =\sum_{a_{3} q_{3} a_{4} q_{4}} \dot{\hat{P}}_{a_{1} q_{1} a_{2} q_{2} a_{3} q_{3} a_{4} q_{4}}\left\langle a_{3} q_{3}|\hat{A}| a_{4} q_{4}\right\rangle \\
& \stackrel{\mathrm{A} .1}{=} \delta_{a_{1} a_{2}} \dot{F}_{a_{2}}\left(q_{2}, T\left(q_{2}, t\right)\right) \sum_{b}\left\langle b q_{1}|\hat{A}| b q_{2}\right\rangle \\
& =\delta_{a_{1} a_{2}} A^{r e d}\left(q_{1}, q_{2}\right) \frac{\partial F_{a_{2}}\left(q_{2}, T\left(q_{2}, t\right)\right)}{\partial T} \dot{T}\left(q_{2}, t\right)
\end{aligned}
$$

where the "reduced part" $A^{\text {red }}\left(q_{1}, q_{2}\right)$ of the matrix $\left\langle a_{1} q_{1}|\hat{A}| a_{2} q_{2}\right\rangle$ is defined by

$$
A^{r e d}\left(q_{1}, q_{2}\right):=\sum_{b_{1} b_{2}}\left\langle b_{1} q_{1}|\hat{A}| b_{2} q_{2}\right\rangle \delta_{b_{1} b_{2}}
$$

The operator $\dot{\hat{P}}(t) \hat{A}$ is thus seen to be diagonal with respect to the quantum numbers of intrinsic excitations and slowly time-dependent as the temperature $T\left(q_{2}, t\right)$ depends slowly on time. The factor $\partial F_{a_{2}}\left(q_{2}, T\left(q_{2}, t\right)\right) / \partial T$ can be reformulated as in Eq. (3.46) and differs from a canonical distribution.

\section{B. Detailed derivation of the Green function (3.40)}

In what follows we show in detail how one obtains the solution (3.40) of the equation (3.32).

$$
\frac{\partial \hat{G}(t, s)}{\partial t}+\frac{i}{\hbar} \hat{Q}(t) \hat{L} \hat{G}(t, s)=\hat{Q}(t) \delta(t-s)
$$

This equation implies that the Green operator $\hat{G}(t, s)$ can be written in the form

$$
\hat{G}(t, s)=\hat{Q}(t) \hat{K}(t, s)
$$

Sunstituting (B.1) into Eq. 3.32

$$
\dot{\hat{Q}}(t) \hat{K}(t, s)+\hat{Q}(t) \frac{\partial \hat{K}(t, s)}{\partial t}+\frac{i}{\hbar} \hat{Q}(t) \hat{L} \hat{Q}(t) \hat{K}(t, s)=\hat{Q}(t) \delta(t-s)
$$

and using the relations (see Appendix $\mathrm{A}$ )

$$
\dot{\hat{Q}}(t)=-\dot{\hat{P}}(t)=-\hat{Q}(t) \dot{\hat{P}}(t),
$$

we find that the Eq. B.2 can be written in the form

$$
\hat{Q}(t)\left[\frac{\partial \hat{K}(t, s)}{\partial t}-\dot{\hat{P}}(t) \hat{K}(t, s)+\frac{i}{\hbar} \hat{L} \hat{Q}(t) \hat{K}(t, s)-\delta(t-s)\right]=0
$$

The operator $\hat{K}(t, s)$ is thus seen to satisfy the equation

$$
\frac{\partial \hat{K}(t, s)}{\partial t}-\hat{W}(t) \hat{K}(t, s)=\delta(t-s)
$$


with

$$
\hat{W}(t):=\dot{\hat{P}}(t)-\frac{i}{\hbar} \hat{L} \hat{Q}(t)
$$

For solving (B.4) in the range $t \geq s$, we put

$$
\hat{K}(t, s)=\theta_{0}(t-s) \hat{\bar{K}}(t, s)
$$

with the initial condition

$$
\hat{\bar{K}}(t, t)=1
$$

Substituting (B.6) into (B.4) yields

$$
\delta(t-s) \hat{\bar{K}}(t, s)+\theta_{0}(t-s)\left\{\frac{\partial \hat{\bar{K}}(t, s)}{\partial t}-\hat{W}(t) \hat{\bar{K}}(t, s)\right\}=\delta(t-s)
$$

and, because of

$$
\delta(t-s) \hat{\bar{K}}(t, s)=\delta(t-s) \hat{\bar{K}}(t, t) \stackrel{\text { B.7 }}{=} \delta(t-s),
$$

we find the homogeneous differential equation

$$
\frac{\partial \hat{\bar{K}}(t, s)}{\partial t}=\hat{W}(t) \hat{\bar{K}}(t, s)
$$

The equation (B.10) and the initial condition (B.7) show that $\hat{\bar{K}}(t, s)$ can be written in the form

$$
\hat{\bar{K}}(t, s)=1+\int_{s}^{t} d \tau \hat{W}(\tau) \hat{\bar{K}}(\tau, s)
$$

The integral equation (B.11) has the solution

$$
\hat{\bar{K}}(t, s)=\hat{T}\left\{e^{\int_{s}^{t} d \tau \hat{W}(\tau)}\right\}
$$

where $\hat{T}$ is the time-ordering operator. The form $(\overline{B .12})$ is thus seen to be a short way to formulate the iterative solution of Eq. (B.11). Finally, from (B.1), (B.6), and (B.12) we find the form

$$
\hat{G}(t, s)=\theta_{0}(t-s) \hat{Q}(t) \hat{T}\left\{e^{\int_{s}^{t} d \tau \hat{W}(\tau)}\right\}
$$

of the Green operator.

\section{Comments on the non-hermitian nature of $\hat{\rho}_{P}(t)$}

As we have already pointed out in Section 3 , our ansatz (3.7) for the approximate form of the density operator is not hermitian. The reader might ask the justified question whether it would not be preferable to derive and solve an equation of motion for the hermitian ansatz

$$
\hat{\rho}_{P}^{h}(t):=\frac{1}{2}\left(\hat{\rho}_{P}(t)+\hat{\rho}_{P}^{\dagger}(t)\right)
$$

rather than solving the equation of motion (3.76) for $\hat{\rho}_{P}(t)$ and subsequently use its hermitian part for the evaluation of physical quantities.

If we use the orthogonal basis functions $\Psi_{a}(q)$ (see Section 2), the matrix representation of $\hat{\rho}_{P}^{h}(t)$ has the form

$$
\left\langle\Psi_{a_{1}}\left(q_{1}\right)\left|\hat{\rho}_{P}^{h}(t)\right| \Psi_{a_{2}}\left(q_{2}\right)\right\rangle=\frac{\delta_{a_{1} a_{2}}}{2}\left(F_{a_{1}}\left(q_{1}, T\left(q_{1}, t\right)\right)+F_{a_{2}}\left(q_{2}, T\left(q_{2}, t\right)\right)\right) R\left(q_{1}, q_{2} ; t\right)
$$


It is seen that (C.2) is close to the matrix representation (3.7) of $\hat{\rho}_{P}(t)$, if the Boltzmann factors $F_{a_{1}}\left(q_{1}, T\left(q_{1}, t\right)\right)$ and $F_{a_{2}}\left(q_{2}, T\left(q_{2}, t\right)\right)$ do not differ very much one from another for pairs of values of the g.c. where the reduced density matrix $R\left(q_{1}, q_{2} ; t\right)$ is substantially different from zero. We expect that the cases where (C.2) differs substantially from the non-hermitian case

$$
\left\langle\Psi_{a_{1}}\left(q_{1}\right)\left|\hat{\rho}_{P}(t)\right| \Psi_{a_{2}}\left(q_{2}\right)\right\rangle=\delta_{a_{1} a_{2}} F_{a_{2}}\left(q_{2}, T\left(q_{2}, t\right)\right) R\left(q_{1}, q_{2} ; t\right)
$$

are more important for small excitation energies (and temperatures), where the canonical ansatz is anyhow less wellfounded.

\section{References}

[1] R. Brown, Phil. Mag. 4, 161 (1828).

[2] A. Einstein, Ann. Phys. (Leipzig) 17, 549 (1905).

[3] H. B. Callen and T. A. Welton, Phys. Rev. 83, 34 (1951).

[4] H. Hofmann, Phys. Rep. 284, 137 (1997) ; "The Physics of Warm Nuclei”, Oxford Univ. Press, 2008.

[5] T. Dittrich, P. Hänggi, G. L. Ingold, B. Kramer, G. Schön, and W. Zwerger, “Quantum Transport and Dissipation”, Wiley-VCH, Weinheim, 1998.

[6] D. Bohm and D. Pines, Phys. Rev. 92, 609 (1953).

[7] U. Brosa and S. Grossmann, Z. Phys. A 310, 177 (1983).

[8] D. L. Hill and J. A. Wheeler, Phys. Rev. 89, 1102 (1953).

[9] J-F. Berger, M. Girod, and D. Gogny, Nucl. Phys. A 428, 23c (1984).

[10] H. Goutte, P. Casoli, J-F. Berger, and D. Gogny, Phys. Rev. C71, 024316 (2005).

[11] N. Dubray, H. Goutte, and J.-P. Delaroche, Phys. Rev. C77, 014310 (2008).

[12] D. Vautherin and D. M. Brink, Phys. Rev. C5, 626 (1972); M. Beiner, H. Flocard, N. Van Giai, and P. Quentin, Nucl. Phys. A238, 29 (1975); E. Chabanat, P. Bonche, P. Haensel, J. Meyer, and R. Schaeffer, Nucl. Phys. A627, 710 (1997); A635, 231 (1998); A643, 441(E) (1998).

[13] D. Gogny, Nucl. Phys. A237, 399 (1975); J. Dechargé and D. Gogny, Phys. Rev. C21, 1568 (1980).

[14] S. Nakajima, Progr. Theor. Phys. 20, 948 (1958).

[15] R. Zwanzig, J. Chem. Phys. 33, 1338 (1960).

[16] P. Ring and P. Schuck, "The Nuclear Many-body Problem”, Springer-Verlag, Berlin, 1980.

[17] N. G. van Kampen, "Stochastic Processes in Physics and Chemistry", North-Holland Publishing Company, 1981.

[18] K. Pomorski, J. Bartel, J. Richert, K. Dietrich, Nucl. Phys. A 605, 87 (1996) (with Refs. to earlier work).

[19] W. J. Swiatecki, Nucl. Phys. A 376, 275 (1982); ibid A 429, 105 (1984).

[20] N. Bohr, Nature 137, 344 (1936) ; Bohr and J. A. Wheeler, Phys. Rev. 56, 426 (1939).

[21] R. Balian, Ann. Phys. Fr. 21, 437 (1996); R. Balian, “From Microphysics to Macrophysics”, Vol. I and II, Springer-Verlag, Berlin, 1991.

[22] D. Agassi, H. A. Weidenmuller and G. Mantzouranis, Phys. Rep. C 22, 145 (1975). 